TRANSACTIONS OF THE

AMERICAN MATHEMATICAL SOCIETY

Volume 365, Number 9, September 2013, Pages 4851-4880

S 0002-9947(2013)05789-3

Article electronically published on April 2, 2013

\title{
REDUCED DIVISORS AND EMBEDDINGS OF TROPICAL CURVES
}

\author{
OMID AMINI
}

\begin{abstract}
Given a divisor $D$ on a tropical curve $\Gamma$, we show that reduced divisors define an integral affine map from the tropical curve to the complete linear system $|D|$. This is done by providing an explicit description of the behavior of reduced divisors under infinitesimal modifications of the base point. We consider the cases where the reduced-divisor map defines an embedding of the curve into the linear system and, in this way, classify all the tropical curves with a very ample canonical divisor. As an application of the reduced-divisor map, we show the existence of Weierstrass points on tropical curves of genus at least two and present a simpler proof of a theorem of Luo on rank-determining sets of points. We also discuss the classical analogue of the (tropical) reduceddivisor map: For a smooth projective curve $C$ and a divisor $D$ of non-negative rank on $C$, reduced divisors equivalent to $D$ define a morphism from $C$ to the complete linear system $|D|$, which is described in terms of Wronskians.
\end{abstract}

\section{INTRODUCTION}

Reduced divisors are one of the main tools in the study of linear systems on tropical curves. Our aim in this paper is to present results on the behavior of reduced divisors under a modification of the base point and on their locus in the underlying complete linear system. After a general introduction on tropical curves and linear systems, providing the basic definitions and results, we show in Section 2 that reduced divisors linearly equivalent to a divisor $D$ define an integral affine map from $\Gamma$ to the complete linear system $|D|$. This is done by providing an explicit description of the behavior of reduced divisors under infinitesimal changes of the base point. We use this description in Section 3 to characterize very ample divisors as the ones defining an embedding of $\Gamma$ in $|D|$ and classify all the tropical curves with a very ample canonical divisor. In Section 4, we present two applications of the reduced divisor map. We first show the existence of tropical Weierstrass points on tropical curves of genus at least two, and second, we derive a simple proof of a theorem of Luo on rank-determining sets of points on tropical curves. In Section 5, we compare the reduced divisor map with maps to tropical projective spaces defined by families of rational functions. Finally, in Section 6 we show that the reduced divisor map has a classical analogue. For a smooth projective curve $C$ and a divisor $D$ of non-negative rank on $C$, reduced divisors equivalent to $D$ define a morphism from $C$ to the complete linear system $|D|$. This map is described in terms of Wronskians.

1.1. Tropical curves and Riemann-Roch theorem. Tropical curves are algebraic curves defined over the tropical semi-ring $\mathbb{T}=(\mathbb{R} \cup\{-\infty\}, \oplus, \odot)$. The tropical

Received by the editors March 9, 2011 and, in revised form, November 22, 2011.

2010 Mathematics Subject Classification. Primary 14T05; Secondary 14C20, 14A10, 05C10.

(C) 2013 American Mathematical Society Reverts to public domain 28 years from publication 4851 
sum $\oplus$ is taking the maximum and the tropical product $\odot$ is the (usual) sum, i.e., $a \oplus b=\max \{a, b\}$ and $a \odot b=a+b$. A (complete) tropical curve is a compact topological space homeomorphic to a one-dimensional simplicial complex equipped with an integral affine structure. This means that the topological space has a finite covering by open sets $U_{i}$ and there are chart maps $\phi_{i}: U_{i} \rightarrow \mathbb{T}^{n_{i}}$, sending $U_{i}$ homeomorphically to a subset $V_{i} \subset \mathbb{T}^{n_{i}}$ such that the change of charts are restrictions of affine maps with integer linear part, i.e., for $i$ and $j$, there exist a matrix $A^{i, j} \in M_{n_{j} \times n_{i}}(\mathbb{Z})$ and an $a \in \mathbb{T}^{n_{j}}$ such that the change of charts $\phi_{j} \phi_{i}^{-1}$ on $\phi_{i}\left(U_{i} \cap U_{j}\right)$ coincides with the map $A . x+a$. It is easy to see that the above definition is equivalent to the following more concrete definition.

A weighted graph $(G, \ell)$ is a finite graph $G=(V, E)$, where each edge $e \in E$ has been assigned a positive length $\ell_{e} \in \mathbb{T}$. This data defines a metric space $\Gamma$, called the geometric representation of $(G, \ell)$, as follows. This is obtained by replacing each edge of the graph by an interval of the given length associated to that edge, the end points of the interval being the corresponding vertices of the original weighted graph. The metric space $\Gamma$ is called a metric graph, and the corresponding weighted graph $(G, \ell)$ is called a model of $\Gamma$. It is clear that different models can give the same metric graph. The choice of a model corresponds to the choice of a finite set of vertices $V$, i.e., a subset of $\Gamma$ such that $\Gamma \backslash V$ is a disjoint union of open intervals. In this paper, when we talk about the vertices of a metric graph, we understand that a model of $\Gamma$ is fixed.

Note that a metric graph $\Gamma$ inherits a topology from the above-described geometric representation and it is possible to speak about the closed and open sets. The distance $\operatorname{dist}_{\Gamma}(v, w)$ between two points $v$ and $w$ of $\Gamma$ is measured in the metric space corresponding to the geometric representation of $\Gamma$ (the subscript $\Gamma$ is omitted if the metric graph $\Gamma$ is clear from the context). A segment in $\Gamma$ is a closed subinterval of one of the intervals in a geometric representation of $\Gamma$ described above.

Each point $v$ of $\Gamma$ has a degree $\operatorname{deg}(v)$. This is defined by taking a sufficiently small neighborhood of $v$ in $\Gamma$, removing $v$ from that neighborhood and counting the number of connected components. Note that every point in the interior of an edge of a model of $\Gamma$ has degree two. Points of degree larger than two are called branching points. The coarsest model for $\Gamma$, if it exists, is a weighted graph in which every vertex has degree different from two, i.e., each vertex is either a branching point or a leaf. Note that the coarsest model is unique if it exists, and it exists if the metric graph is connected and there is at least one branching point, i.e., if the metric graph is not a circle (a weighted graph is allowed to contain loops).

An abstract tropical curve is a metric graph where some of the edges incident with vertices of degree one (leaves) have infinite length. Such edges are identified with the interval $[0, \infty]$, such that $\infty$ is identified with the vertex of degree one, and are called infinite ends or bounds. Since infinite ends do not play any interesting role with respect to divisor theory on a tropical curve, in this paper we suppose that $\Gamma$ does not have any infinite end.

The genus of a tropical curve $\Gamma$, denoted by $g(\Gamma)$ or $g$, is the first Betti number of $\Gamma$ as a topological space. Given a model $(G, \ell)$ of $\Gamma$ with $G=(V, E)$, the genus of $\Gamma$ is $g=|E|-|V|+1$.

A divisor $D$ on a tropical curve $\Gamma$ is an element of the infinite free abelian group $\operatorname{Div}(\Gamma)$ on points of $\Gamma$. The basis element of $\operatorname{Div}(\Gamma)$ corresponding to a point $v$ of $\Gamma$ is denoted by $(v)$. Each divisor $D \in \operatorname{Div}(\Gamma)$ can be uniquely written as 
$D=\sum_{v \in \Gamma} a_{v}(v)$ with $a_{v} \in \mathbb{Z}$, and all but a finite number of $a_{v}$ 's are zero. The coefficient $a_{v}$ of $(v)$ in $D$ is denoted by $D(v)$. A divisor $D$ is called effective if $D \geq 0$, i.e., if $D(v) \geq 0$ for all $v \in \Gamma$. The degree of a divisor $D \in \operatorname{Div}(\Gamma)$, denoted by $\operatorname{deg}(D)$, is defined as $\operatorname{deg}(D):=\sum_{v \in \Gamma} D(v)$. The canonical divisor of a tropical curve $\Gamma$ is the divisor $K_{\Gamma}$ defined by

$$
K_{\Gamma}:=\sum_{v \in \Gamma}(\operatorname{deg}(v)-2)(v) .
$$

A rational function on a tropical curve $\Gamma$ is a continuous piecewise linear function $f: \Gamma \rightarrow \mathbb{R} \cup\{ \pm \infty\}$ with integral slopes at every point and such that the number of linear parts of $f$ on every edge is finite and the only points $v$ with $f(v)= \pm \infty$ are unbounded ends. (This is simply equivalent to a continuous map respecting the integral affine structure of $\Gamma$ and the obvious integral affine structure on $\mathbb{R} \cup$ $\{ \pm \infty\}$.) The order $\operatorname{ord}_{f}(v)$ of a rational function $f$ on $\Gamma$ at a point $v$ is the sum of the outgoing slopes of $f$ along the segments of $\Gamma$ emanating from $v$. Given a rational function $f$, the $\operatorname{divisor} \operatorname{div}(f)=\sum_{v} \operatorname{ord}_{v}(f)(v)$ is called the principal divisor associated to $f$. Note that if $v$ is not a branching point of $\Gamma$ and the function $f$ does not change its slope at $v$, then $\operatorname{ord}_{f}(v)=0$; thus, there are only finitely many points $v$ with $\operatorname{ord}_{f}(v) \neq 0$ and $\operatorname{div}(f) \in \operatorname{Div}(\Gamma)$. The degree of a principal divisor is equal to zero since each linear part of a given slope in a rational function contributes twice in the degree, once with a positive sign and once with a negative sign. Two divisors $D$ and $D^{\prime}$ on $\Gamma$ are called linearly equivalent, written $D \sim D^{\prime}$, if there exists a rational function $f$ on $\Gamma$ such that $D=D^{\prime}+\operatorname{div}(f)$.

Let $D$ be a divisor on a tropical curve $\Gamma$. The (complete) linear system of $D$, denoted by $|D|$, is defined as the set of all effective divisors $E$ linearly equivalent to $D$,

$$
|D|:=\{E \in \operatorname{Div}(\Gamma): E \geq 0 \text { and } E \sim D\} .
$$

The rank of a divisor $D \in \operatorname{Div}(\Gamma)$, denoted by $r(D)$, is defined by

$$
r(D):=\min _{E: E \geq 0,|D-E|=\emptyset} \operatorname{deg}(E)-1 .
$$

Gathmann and Kerber [5] and, independently, Mikhalkin and Zharkov [9] proved the following Riemann-Roch theorem for tropical curves (see also [7] for another more combinatorial proof of this theorem, similar to the proof of Baker and Norine's Riemann-Roch theorem for graphs [3] ).

Theorem 1 (Tropical Riemann-Roch). Let $D$ be a divisor on a tropical curve $\Gamma$ of genus $g$ and $K$ be the canonical divisor of $\Gamma$. Then

$$
r(D)-r(K-D)=\operatorname{deg}(D)+1-g .
$$

1.2. Reduced divisors. The proof of the Riemann-Roch theorem for graphs and tropical curves is based on the notion of reduced divisors that we now explain. (We refer to [1] for a different, more geometric, proof of a slightly more general result in the graph case.)

Let $\Gamma$ be a tropical curve and $v_{0}$ be a given (base) point of $\Gamma$. For a closed connected subset $X$ of $\Gamma$ and a point $v \in \partial X$, the number of edges leaving $X$ at $v$, denoted by $\operatorname{deg}_{X}^{\text {out }}(v)$, is by definition the maximum size of a collection of internally disjoint segments in $\Gamma \backslash(X-\{v\})$ with an end in $v$. In what follows we also call such a connected subset of $\Gamma$ a cut in $\Gamma$. A boundary point $v$ of a closed connected subset $X$ of $\Gamma$ is called saturated with respect to $D \in \operatorname{Div}(\Gamma)$, and the cut $X$ if the 
number of edges leaving $X$ at $v$ is at most $D(v)$ (i.e., if $\left.\operatorname{deg}_{X}^{\text {out }}(v) \leq D(v)\right)$ and is called non-saturated, otherwise. A cut is said to be saturated if all its boundary points are saturated. When talking about saturated and non-saturated points, we sometimes omit $D$ and/or the set $X$ if they are clear from the context.

A divisor $D$ on a tropical curve $\Gamma$ is said to be $v_{0}$-reduced if the following two properties are satisfied:

- For all points $v \neq v_{0}$ of $\Gamma, D(v) \geq 0$; in other words, if all the coefficients of $D$ are non-negative except possibly for the base point $v_{0}$.

- For every closed connected subset $X$ of points of $\Gamma$ which does not contain $v_{0}$, there exists a non-saturated point $v \in \partial X$.

Every divisor is equivalent to a unique $v_{0}$-reduced divisor. Here we provide a quick proof and refer to [3, 7] for more details.

Theorem 2. Let $\Gamma$ be a tropical curve and $v_{0}$ be a point of $\Gamma$. For every divisor $D \in \operatorname{Div}(\Gamma)$, there exists a unique $v_{0}$-reduced divisor $D_{0}$ such that $D_{0} \sim D$.

Proof. Let $\mathcal{T}_{D}$ be the set of all divisors $D^{\prime} \sim D$ such that all the coefficients of $D^{\prime}$ are non-negative at every point of $\Gamma$ except possibly at $v_{0}$, and such that, in addition, the coefficient of $v_{0}$ is maximum possible with respect to this property. Note that $\mathcal{T}_{D}$ is non-empty. This is simply because there are linearly equivalent divisors to $D$ with non-negative coefficients at every point $v \neq v_{0}$ of $\Gamma$. To see this, note that if $k$ is large enough (as a function of the number of branching points, the diameter of the underlying graph, and the maximum absolute value of the coefficients of $D$ ), then it can be easily shown that the divisor $D+k\left(v_{0}\right)$ is equivalent to an effective divisor $E$. Now $E-k\left(v_{0}\right) \sim D$ has the required property. Note that the Riemann-Roch theorem implies that if $k$ is large enough so that the degree of $D+k\left(v_{0}\right)$ is larger than $g$, then the rank of $D+k\left(v_{0}\right)$ is non-negative; however the existence of a large enough $k$ with this property does not need the full strength of the Riemann-Roch theorem (whose proof is based on reduced divisors).

We next observe that $\mathcal{T}_{D}$ inherits a natural topology from the topology of $\Gamma$ and is compact.

Let $N$ be the sum of the coefficients of points of $\Gamma \backslash\left\{v_{0}\right\}$ in any divisor in $\mathcal{T}_{D}$ (by the definition of $\mathcal{T}_{D}, N$ is well defined). Let $\mathcal{A}$ be the subset of $\mathbb{R}^{N}$ defined as follows:

$$
\mathcal{A}:=\left\{\left(x_{1}, \ldots, x_{N}\right) \mid 0 \leq x_{1} \leq x_{2} \leq \cdots \leq x_{N}\right\} .
$$

Note that $\mathcal{A}$ has a natural total order defined by the lexicographical order: $\left(x_{1}, \ldots\right.$, $\left.x_{N}\right)<\left(y_{1}, \ldots, y_{N}\right)$ if and only if there exists a non-negative integer $i$ such that $x_{1}=y_{1}, \ldots, x_{i}=y_{i}$ and $x_{i+1}<y_{i+1}$.

Define a continuous map $F: \mathcal{T}_{D} \rightarrow \mathcal{A}$ as follows: for each divisor $D^{\prime} \in \mathcal{T}_{D}$, consider the multiset $A\left(D^{\prime}\right)$ of points in $\Gamma \backslash\left\{v_{0}\right\}$, where each point $v \neq v_{0}$ appears in this multiset exactly $D^{\prime}(v)$ times. Define $F\left(D^{\prime}\right)$ to be the point of $\mathcal{A}$ defined by the multiset of distances $\operatorname{dist}_{\Gamma}\left(v, v_{0}\right)$ for $v \in A\left(D^{\prime}\right)$, ordered in an increasing way to define a point of $\mathcal{A}$. It is straightforward to show that the map $F$ is continuous.

Since $\mathcal{T}_{D}$ is a compact set and $F$ is continuous, there exists a divisor $D_{0}$ such that $F$ takes its minimum value on $D_{0}$, i.e., $F\left(D_{0}\right)=\min _{D^{\prime} \in \mathcal{T}_{D}} F\left(D^{\prime}\right)$. We claim that $D_{0}$ is $v_{0}$-reduced. The first property is clearly verified by the choice of $D_{0}: D_{0} \sim D$ and the coefficients $D_{0}(v)$ are all non-negative for $v \neq v_{0}$ in $\Gamma$. We must show that every connected set $X$ which does not contain $v_{0}$ has a non-saturated point on its boundary. For the sake of a contradiction, suppose this is not the case and let 
$X$ be the connected set violating this condition. This means that for all $v \in \partial X$, $D_{0}(v) \geq \operatorname{deg}_{X}^{\text {out }}(v)$. By the definition of $\operatorname{deg}_{X}^{\text {out }}$, there exists an $\epsilon>0$ such that for each vertex $v \in \partial X$, there exist closed segments $I_{1}^{v}, \ldots, I_{\operatorname{deg}_{X}^{\text {out }}(v)}$ emanating from $v$ with the following properties:

- For $v \in \partial X$ and $1 \leq j \leq \operatorname{deg}_{X}^{\text {out }}(v)$, each $I_{j}^{v}$ has length $\epsilon$, and the half-open line segments $I_{j}^{v} \backslash v$ live outside $X$ and are all disjoint.

Now define the rational function $f: \Gamma \rightarrow \mathbb{R}$ as follows: $f$ takes value zero on $X$, is linear of slope -1 on each interval $I_{j}^{v}$ and takes value $-\epsilon$ on all the points of $\Gamma \backslash\left(X \cup \cup I_{j}^{v}\right)$, the union being over all $v \in \partial X$ and $1 \leq j \leq \operatorname{deg}_{X}^{\text {out }}(v)$. Since $\operatorname{deg}_{X}^{\text {out }}(v) \leq D_{0}(v)$, the divisor $D_{0}+\operatorname{div}(f)$ lies inside $\mathcal{T}_{D}$. Since the distance of $X$ to $v_{0}$ is strictly larger than the distance from the set $X \cup \cup I_{j}^{v}$ to $v_{0}$, and the boundary points of $X$ are all in the multiset $A\left(D_{0}\right)$, it is straightforward to see that $F\left(D_{0}\right)>F\left(D_{0}+\operatorname{div}(f)\right)$, and this contradicts the choice of $D_{0}$. This proves the existence.

To prove the uniqueness, for the sake of a contradiction, suppose there are two $v_{0}$-reduced divisors $D_{0}$ and $D_{0}^{\prime}$ which are linearly equivalent. This means that there exists a non-constant rational function $f$ such that $D_{0}^{\prime}=D_{0}+\operatorname{div}(f)$. Since $f$ is not a constant function, we can assume that $f$ does not take its maximum at $v_{0}$ (if not, we change the role of $D_{0}$ and $D_{0}^{\prime}$, and replace $f$ by $-f$ ). Let $X$ be a connected component of the set of all points where $f$ takes its maximum. Note that $v_{0} \notin X$. For any point $v$ lying on the boundary of $X$, the slope of $f$ along any segment $I_{j}^{v}$ emanating from $v$ is at most -1 . It follows that the coefficient of $(v)$ in $D_{0}+\operatorname{div}(f)$ is at most $D_{0}(v)-\operatorname{deg}_{X}^{\text {out }}(v)$. Since $D_{0}$ is $v_{0}$-reduced, there exists a point $v \in \partial X$ such that $D(v)<\operatorname{deg}_{X}^{\text {out }}(v)$. For this point, $D_{0}^{\prime}(v)=D_{0}(v)-\operatorname{deg}_{X}^{\text {out }}(v)<0$, which contradicts the assumption that $D_{0}^{\prime}$ is non-negative outside $v_{0}$. The uniqueness follows.

\section{The map Red From $\Gamma$ to A (COMPlete) Linear System}

Let $D$ be a divisor and $|D|$ its complete linear system. Suppose that $r(D) \geq 0$ such that $|D|$ is non-empty. There is a natural polyhedral structure on $|D|$ that we now explain.

Let $\Gamma$ be a tropical curve and $N$ be an integer. The symmetric product $\Gamma^{(N)}$ is defined as the set of all unordered $N$-tuples of points of $\Gamma$, i.e.,

$$
\Gamma^{(N)}:=\Gamma^{N} / \mathfrak{S}_{N},
$$

where $\mathfrak{S}_{N}$ is the symmetric group of degree $N$ and $\Gamma^{N}$ is the product of $N$ copies of $\Gamma$.

The linear system $|D|$ is naturally a subset of the symmetric product $\Gamma^{(\operatorname{deg}(D))}$ : define a map $\iota:|D| \hookrightarrow \Gamma^{(\operatorname{deg}(D))}$ by sending each $E \in|D|$ to $\iota(E)$ of $\Gamma^{(\operatorname{deg}(D))}$ consisting of the (unordered) multiset of all the points $v$ with $E_{v} \neq 0$. The topology of $|D|$ is the natural topology induced from $\Gamma^{(\operatorname{deg}(D))}$. We will explain the cell structure of $|D|$ in more detail in the next section.

2.1. The integral affine structure on $\Gamma^{(N)}$ and $|D|$. We start this section by describing a natural integral affine structure on $\Gamma^{(\operatorname{deg}(D))}$ induced from $\Gamma$. First, we need to say more precisely what we mean by an integral affine structure. Recall 
that a manifold with an integral affine structure of dimension $n$ is given by the following data:

- A (finite) collection of open sets (charts) $U_{i}$ covering all of $X$.

- A homeomorphism $\phi_{i}$ from $U_{i}$ to an open subset $V_{i}$ of $\mathbb{R}^{n}$ for each $i$ such that for different $i$ and $j$ the change of chart map $\phi_{j} \phi_{i}^{-1}: \phi_{i}\left(U_{j} \cap U_{i}\right) \rightarrow$ $\phi_{j}\left(U_{i} \cap U_{j}\right)$ is the restriction of an integral affine map from $\mathbb{R}^{n}$ to $\mathbb{R}^{n}$, i.e., there exists $a \in \mathbb{R}^{n_{j}}$ and $A \in M_{n \times n}(\mathbb{Z})$ such that $\phi_{i} \phi_{j}^{-1}(x)=A x+a$ for all $x \in \phi_{i}\left(U_{j} \cap U_{i}\right)$.

Since the objects we will be considering will have singularities, we need to modify the above definition to also cover the case of stratified manifolds. Roughly speaking, if $X$ admits a filtration $X_{0} \subset X_{1} \subset \cdots \subset X_{n-1} \subset X_{n}=X$ such that for each $i$, $X_{i} \backslash X_{i-1}$ is a (possibly disconnected) manifold of dimension $i$, then it is natural to impose that each $X_{i} \backslash X_{i-1}$ is an $i$-dimensional manifold with an integral affine structure and such that, in addition, there is a compatibility between the integral affine structure on $X_{i} \backslash X_{i-1}$ and the one "induced" from $X_{i+1} \backslash X_{i}$ on $X_{i} \backslash X_{i-1}$. To give a more precise meaning to this, in what follows it turns out to be more convenient for our purpose to work with abstract rational polyhedral complexes (which will carry by definition an integral affine structure in the above general sense).

Let $N$ be a lattice of rank $n$ in the $n$-dimensional real vector space $N_{\mathbb{R}}=N \otimes_{\mathbb{Z}} \mathbb{R}$. Recall that a rational polyhedral complex $\Sigma$ in $N_{\mathbb{R}}$ is a finite collection of (convex) rational polyhedra in $N_{\mathbb{R}}$ which satisfies the following two properties:

- If $\sigma \in \Sigma$ and $\tau$ is a face of $\sigma$, then $\tau$ is in $\Sigma$.

- If $\sigma$ and $\tau$ are in $\Sigma$, then $\sigma \cap \tau$ is a (possibly empty) common face of $\sigma$ and $\tau$ (which is also in $\Sigma$ ).

Since the polyhedral complex $\Sigma$ is rational, for each point $x$ in the relative interior of a polyhedra $\sigma \in \Sigma$, the tangent space of $x$ in $\sigma$ has a canonical sublattice of full rank. This canonically coincides with the sublattice $N_{\sigma}$ of $N$ of dimension $\operatorname{dim}(\sigma)$ defined by the set of all points of $N$ which lie on the rational plane $\operatorname{span}(\sigma-$ $x)=: N_{\sigma, \mathbb{R}}$ in $N_{\mathbb{R}}$ defined by $\sigma$. Under this identification, one canonically has an isomorphism between the tangent space of the interior of $\sigma$ with $\operatorname{int}(\sigma) \times N_{\sigma, \mathbb{R}}$.

Now to make the above definition independent of the embedding into a fixed $N_{\mathbb{R}}$, we can proceed as follows. An abstract rational polyhedral complex structure $\Sigma$ on a Hausdorff topological space $X$ is a finite collection of closed topological subspaces $\sigma \subseteq X$ with the following properties. Each $\sigma$ is homeomorphic to a full-dimensional polyhedra $|\sigma|$ in a real vector space $N_{\sigma, \mathbb{R}}$. In addition, $N_{\sigma, \mathbb{R}}$ has a sublattice of full rank $N_{\sigma}$ such that $|\sigma|$ is rational with respect to $N_{\sigma}$. By rational, we mean that the real vector space defined by any face of $|\sigma|$ comes (by extension of scalars from $\mathbb{Q}$ to $\mathbb{R}$ ) from a subvector space of $N_{\sigma, \mathbb{Q}}=N \otimes_{\mathbb{Z}} \mathbb{Q}$. Under these homeomorphisms, $\Sigma$ satisfies the following properties, analogous to the properties of a rational polyhedral complex in $N_{\mathbb{R}}$ given above. For $\sigma \in \Sigma$, define $F_{\sigma}$ as the poset (under inclusion) of all $\tau \in \Sigma$ such that $\tau \subseteq \sigma$. For convenience, assume that $\emptyset \in F_{\sigma}$ for all $\sigma \in \Sigma$.

- If $\sigma \in \Sigma$, there is an isomorphism of posets from $F_{\sigma}$ to the face poset of $|\sigma|$. Under this isomorphism, if $\tau \in F_{\sigma}$ and $\eta$ is the corresponding face of $|\sigma|$ in $N_{\sigma, \mathbb{R}}$, there exists an isomorphism of rational polyhedra between $|\tau|$ and $\eta$. - If $\sigma$ and $\tau$ are in $\Sigma$, then $\sigma \cap \tau$ is in $\Sigma$ and thus belongs to both $F_{\sigma}$ and $F_{\tau}$. 
Given an abstract rational polyhedral complex structure $\Sigma$ on $X$, define $X_{i}$ as the union of all $\sigma \in \Sigma$ such that $|\sigma|$ has dimension $i$. The following facts are easy to verify:

- For each $i, X_{i} \backslash X_{i-1}$ is naturally an (open) manifold of dimension $i$ with an integral affine structure. More precisely, $X_{i} \backslash X_{i-1}$ is the disjoint union of the relative interior of all $\sigma \in \Sigma$ such that $|\sigma|$ has dimension $i$.

- If $\Sigma$ and $\Sigma^{\prime}$ are abstract rational polyhedral structures on $X$ and $X^{\prime}$, respectively, there exists a natural abstract rational polyhedral complex structure $\Sigma \times \Sigma^{\prime}$ on $X \times X^{\prime}$. The elements of $\Sigma \times \Sigma^{\prime}$ are of the form $\sigma \times \sigma^{\prime} \subset X \times X^{\prime}$ for $\sigma \in \Sigma$ and $\sigma^{\prime} \in \Sigma^{\prime}$.

Any metric graph $\Gamma$ with a simple model $G=(V, E)$ admits an abstract rational polyhedral structure given by $\Sigma=V \cup E$. (Recall that $G$ is called simple if it does not have any loop or parallel edges.) For each edge $e \in E$, identify $e$ with the interval $[0, \ell(e)]$ of length $\ell(e)$ in $\mathbb{R}$ and let $N_{e}=\mathbb{Z} \subset \mathbb{R}$. This naturally defines an abstract rational polyhedral complex structure $\Sigma^{N}=\Sigma_{G=(V, E)}^{N}$ on $\Gamma^{N}$. Each $\sigma \in \Sigma_{G=(V, E)}$ is of the form $\sigma_{1} \times \cdots \times \sigma_{N}$ for $\sigma_{i} \in V \cup E$. Note that the integral affine structure on $\Gamma^{N}$ is invariant under the action of the symmetric group $\mathfrak{S}_{N}$. Also note that subdividing the rational polyhedral structure of $\Gamma$ consists of choosing another model $G^{\prime}=\left(V^{\prime}, E^{\prime}\right)$ of $\Gamma$ which is a refinement of $G$ (i.e., $\left.V \subseteq V^{\prime}\right)$. This in turn induces a subdivision of the abstract rational polyhedral structure of $\Gamma^{N}$.

Next we show that the topological quotient $\Gamma^{(N)}$ of $\Gamma^{N}$ by $\mathfrak{S}_{N}$ admits an integral affine structure given by an abstract rational polyhedral complex structure $\Sigma^{(N)}$. In addition, the projection map $\pi: \Gamma^{N} \rightarrow \Gamma^{(N)}$ is "integral affine" in the sense that for all $x$ in the relative interior of a $\sigma$ in $\Sigma^{N}$, with image $y$ in the relative interior of $\eta$ in $\Sigma^{(N)}, N_{\eta} \subseteq \pi_{*}\left(N_{\sigma}\right)$ ( $\pi_{*}$ is the push forward of tangent vectors from $\Gamma^{N}$ to $\left.\Gamma^{(N)}\right)$.

To describe the integral affine structure of $\Gamma^{(N)}$, we fix a model $G=(V, E)$ of $\Gamma$ without loops and parallel edges. From the description below, it will be clear that choosing another simple model $G^{\prime}=\left(V^{\prime}, E^{\prime}\right)$ of $\Gamma$ which is a refinement of $G$ consists of subdividing the abstract rational polyhedral structure of $\Gamma^{(N)}$, and thus, the induced integral affine structure does not depend on the particular choice of the model.

Let $v_{1}, \ldots, v_{n}$ be the set of all the vertices of $V$. The enumeration defines an orientation on the set of edges of $\Gamma$, thus a lexicographical order on the points of the edge respecting the orientation, and also a lexicographical order on the edges set. Since $\Gamma$ does not have any loop or parallel edges, there is no ambiguity in the definition of the lexicographical order. First, for an edge $e$ of $G$ of length $\ell(e)$ and an integer $m$, consider the closed subset $e^{(m)}=e^{m} / \mathfrak{S}_{m}$ of $\Gamma^{(m)}$ and identify $\left|e^{(m)}\right|$ with the rational polytope in $\mathbb{R}^{m}$ consisting of all the points $\left(x_{1}, \ldots, x_{m}\right)$ such that $0 \leq x_{1} \leq x_{2} \leq \cdots \leq x_{m} \leq \ell(e)$. The faces of $\left|e^{(m)}\right|$ induce a decomposition of $e^{(m)}$ into closed subsets and define a rational polyhedral structure on $e^{(m)}$. Now, more generally, given a sequence $\underline{e}$ consisting of $N$ edges $e_{1}=e_{1}=\cdots=e_{1}<e_{2}=\cdots=$ $e_{i}<\cdots<e_{k}=\cdots=e_{k}$ of $\Gamma$ ordered in the lexicographical order, and in which each edge $e_{i}$ is repeated $s_{i}$ times with $\sum_{i=1}^{k} s_{i}=N$, consider the closed subset $\sigma_{\underline{e}}$ of $\Gamma^{(N)}$ defined by $\sigma_{\underline{e}}=e_{1}^{\left(s_{1}\right)} \times \cdots \times e_{k}^{\left(s_{k}\right)}$ and consider its geometric realization as a rational polytope in $\mathbb{R}^{N}$ defined by

$$
\left|\sigma_{\underline{e}}\right|=\left|e_{1}^{\left(s_{1}\right)}\right| \times \cdots \times\left|e_{k}^{\left(s_{k}\right)}\right| .
$$


The faces of $\left|\sigma_{\underline{e}}\right|$ define a decomposition of $\sigma_{\underline{e}}$ into closed subsets and induce an abstract rational polyhedral structure $\Sigma_{\underline{e}}$ on $\sigma_{\underline{e}}$. Note that the relative interior $\operatorname{int}\left(\sigma_{\underline{e}}\right)$ of $\sigma_{\underline{e}}$ is given by

$$
\begin{aligned}
& \operatorname{int}\left(\sigma_{\underline{e}}\right)=\left\{\left(x_{1}, \ldots, x_{s_{1}}, x_{s_{1}+1}, \ldots, x_{s_{1}+s_{2}}, \ldots, x_{s_{1}+\cdots+s_{k-1}+1}, \ldots, x_{N}\right) \in \Gamma^{N}\right. \\
& x_{1}<\cdots<x_{s_{1}} \in \operatorname{int}\left(e_{1}\right) \\
& x_{s_{1}+1}<\cdots<x_{s_{1}+s_{2}} \in \operatorname{int}\left(e_{2}\right) ; \ldots \text {; } \\
& \left.x_{s_{1}+\cdots+s_{k-1}+1}<\cdots<x_{N} \in \operatorname{int}\left(e_{k}\right)\right\} \text {. }
\end{aligned}
$$

Here int $(e)$ denotes the interior of an edge $e$. The abstract rational polyhedral structure $\Sigma$ on $\Gamma^{(N)}$ is defined as follows. $\Sigma^{(N)}$ is the union of all the closed subsets of $\Gamma^{(N)}$ which appear in some $\Sigma_{\underline{e}}$ for some sequence of edges $\underline{e}, \Sigma^{(N)}=\bigcup_{\underline{e}} \Sigma_{\underline{e}}$. Each element $\tau$ of $\Sigma^{(N)}$ belongs to some $\Sigma_{e}$, and thus one can define $|\tau|$ and its rational polyhedral structure to be the one defined by the corresponding face of $\left|\sigma_{\underline{e}}\right|$. It is straightforward to check that this does not depend on the choice of the sequence $\underline{e}$, and thus one obtains a global structure of an abstract rational polyhedral complex on $\Gamma^{(N)}$. This abstract rational polyhedral structure on $\Gamma^{(N)}$ is pure of dimension $N$, i.e., all the maximal cells in $\Sigma^{(N)}$ have dimension $N$.

Note that in the stratification $X_{0} \subset X_{1} \subset \cdots \subset X_{N}$ of $\Gamma^{(N)}$ defined by $\Sigma^{(N)}$, $X_{N} \backslash X_{N-1}$ is the disjoint union of all the open sets $\operatorname{int}\left(\sigma_{\underline{e}}\right)$. If among the strict inequalities describing $\operatorname{int}\left(\sigma_{\underline{e}}\right) i$ of them become equality (also counting the situations where the points lie on the boundary of an edge), then these so-defined cells become disjoint and their union forms the subset $X_{N-i} \backslash X_{N-i-1}$. It is clear that the set of vertices $X_{0}$ consists of all the $N$-tuples $v_{i_{1}}, \ldots, v_{i_{N}}$ of vertices of $G$ with $1 \leq i_{1} \leq \cdots \leq i_{N} \leq n$.

Our next aim will be to explain how $|D|$ inherits a natural structure of an abstract rational polyhedral complex from that of $\Gamma^{(\operatorname{deg}(D))}$. This essentially amounts to providing the description of the cell structure on $|D|$ given in [5, 9] and 6]. We point out that this cell structure is rational, is induced by the rational polyhedral complex structure of $\Gamma^{(\operatorname{deg}(D))}$, and is so that the inclusion map $|D| \hookrightarrow \Gamma^{(\operatorname{deg}(D))}$ is integral affine.

Let $\Gamma$ be a tropical curve with a simple model $G=(V, E)$. Let $v_{1}, \ldots, v_{n}$ be the set of all the vertices of $V$. Let $D$ be a divisor of degree $d=\operatorname{deg}(D)$ of nonnegative rank. Consider the abstract rational polyhedral complex structure $\Sigma^{(d)}$ on $\Gamma^{(d)}$ described above. For each $\tau \in \Sigma^{(d)}$, the intersection of $|D|$ with the relative interior of $\tau$ has a finite number of connected components. Define $\Sigma_{D}$ as the family of all the closed subsets of $|D|$ which are the closure in $\Gamma^{(d)}$ (or equivalently in $|D|$ ) of a connected component of $|D| \cap \operatorname{int}(\tau)$ for a $\tau \in \Sigma^{(d)}$. We claim that $\Sigma_{D}$ defines an abstract rational polyhedral structure on $|D|$. This is reduced to proving two claims, namely that first, for each $\eta$ the closure of a connected component of $|D| \cap \operatorname{int}(\tau)$, the geometric realization $|\eta|$ in $|\tau|$ is a rational polytope, and that, in addition, each face of $|\eta|$ is the geometric realization of an element $\gamma$ in $\Sigma_{D}$, this second assertion itself being a direct consequence of the first one and the assertion that the dimension of $|D| \cap \operatorname{int}(\tau)$ is locally constant (thus, $\eta$ cannot have a proper face in the interior of $\tau$ ). 
Recall that the enumeration of the vertices induces an orientation of the edges of $\Gamma$ and a lexicographical order on the edges. The relative interior of an element $\tau \in \Sigma^{(d)}$ is the set of all the points $\underline{x}=\left\{x_{1}, \ldots, x_{d}\right\}$ of $\Gamma^{(d)}$ described by

- A map $\mathfrak{d}: V \sqcup E \rightarrow \mathbb{Z}$ which encodes the following data: For any vertex $v \in V, \mathfrak{d}(v)$ is the number of $x_{i}$ in $\underline{x}$ which are equal to $v$. For any edge $e \in E, \mathfrak{d}(e)$ is the number of $x_{i}$ in $\underline{x}$ which lie in the interior of the edge $e$. Note that $\sum_{v \in V} \mathfrak{d}(v)+\sum_{e \in E} \mathfrak{d}(e)=d$.

- In addition, for every edge $e$, an ordered decomposition of $\mathfrak{d}(e)=\mathfrak{d}_{1}(e)+$ $\cdots+\mathfrak{d}_{s_{e}}(e)$ for some non-negative integer $s_{e}=s_{e}(\tau)$. This is given by looking at the location of all the points $x_{i}$ which are in the interior of the edge $e$ : among these points, the first $\mathfrak{d}_{e}(1)$ are the same and are strictly smaller than the following $\mathfrak{d}_{2}(e)$ points which are the same, these points being strictly smaller than the following $\mathfrak{d}_{3}(e)$ points which are the same, and so on.

We now claim that $\operatorname{int}(\tau) \cap|D|$ is a disjoint union of rational polyhedra in $N_{\tau, \mathbb{R}}$ of the same dimension. This establishes the two above-mentioned assertions and thus shows that $|D|$ has an abstract rational polyhedral complex structure.

To see this, first note that $|D| \cap \operatorname{int}(\tau)$ consists of all the divisors $D^{\prime}$ linearly equivalent to $D$ such that

- the coefficient of $D^{\prime}$ at $v$ is equal to $\mathfrak{d}(v)$, and

- for every edge $e$, there are points $x_{1}^{e}<x_{2}^{e}<\cdots<x_{s_{e}}^{e} \in \operatorname{int}(e)$ such that the support of $D^{\prime}$ in the interior of $e$ is given by $\sum \mathfrak{d}_{i}(e)\left(x_{i}^{e}\right)$.

To describe the rational polyhedral structure of $|D| \cap \operatorname{int}(\tau)$, one also has to fix the slopes of the rational function $f$ which provides the linear equivalence of $D$ and $D^{\prime}$, i.e., $\operatorname{div}(f)+D=D^{\prime}$, at the beginning of any edge $e$. In other words, one fixes an integer-valued function $m: E \rightarrow \mathbb{Z}$ and assumes that $f$ has slope equal to $m(e)$ along $e$ at the starting point of $e$. The combinatorial data $\mathfrak{d}(),. \mathfrak{d}_{i}($.$) and m($.$) provide$ linear equations and inequalities with rational coefficients which define a rational polyhedral part of $|D| \cap \operatorname{int}(\tau)$ [5]. In addition, by the up-to-an-additive-constant uniqueness of the rational function $f$, it becomes immediately clear that these polyhedra form different connected components of $|D| \cap \operatorname{int}(\tau)$. To show the assertion that the dimensions are all equal, let $n_{\tau}$ be the number of connected components of the topological space obtained by removing $s_{e}$ points from the interior of any edge $e$. Note that for any $D^{\prime}=\sum_{v \in V} \mathfrak{d}(v)(v)+\sum_{e \in E} \sum_{i=1}^{s_{e}} \mathfrak{d}_{i}(e)\left(x_{i}^{e}\right) \in|D| \cap \operatorname{int}(\tau)$, the number of connected components of $\Gamma \backslash\left\{x_{i}^{e}\right\}_{i, e}$ is $n_{\tau}$. Each connected component $A$ of $\Gamma \backslash\left\{x_{i}^{e}\right\}_{i, e}$ forms a saturated cut in $\Gamma$, because all the points on the boundary have out-going degree one and coefficient at least one. For small enough positive $\epsilon$, by considering small out-going segments $I_{\epsilon}^{v}$ of length $\epsilon / D^{\prime}(v)$ from $A$ at $v$ for any point $v \in \partial A$, one can define a rational function $f_{\epsilon}$ with value zero at $A$, with slope $-D^{\prime}(v)$ along the segment $I_{\epsilon}^{v}$, and with value $-\epsilon$ outside the union of $A$ and the segments $I_{\epsilon}^{v}$. In this way, by combining these functions, one can show that the $n_{\tau}$ connected components of $\Gamma \backslash\left\{x_{i}^{e}\right\}_{i, e}$ allow one to infinitesimally move $D^{\prime}$ in an open subset of dimension $n_{\tau}-1$ in $|D| \cap \operatorname{int}(\tau)$, which shows that the dimension of each connected component of $|D| \cap \operatorname{int}(\tau)$ is $n_{\tau}-1$; cf. [6] and Proposition 22.

It can happen in general that $|D|$ is not pure dimensional, i.e., some maximal cells of $|D|$ can have dimension less than the dimension of $|D|$. For example, if the tropical curve consists of four points $P, Q, R, S$ with an edge between $P$ and $Q$, two parallel edges from $P$ to $R$ and two parallel edges from $Q$ to $S$, and the divisor $D$ 
is equal to $P+Q$, the complete linear system $|D|$ consists of three maximal cells: two segments and a triangle.

2.2. The reduced divisor map. Let $D$ be a divisor with $r(D) \geq 0$. For a point $P \in \Gamma$, we denote by $D_{P}$ the unique $P$-reduced divisor linearly equivalent to $D$. Note that $r(D) \geq 0$ is equivalent to $D_{P} \geq 0$ for every point $P$. Since all the coefficients of $D_{P}$ are already non-negative, this simply means that the coefficient $D_{P}(P)$ of $D_{P}$ at $P$ is non-negative.

Before stating the main theorem of this section, let us recall that a map $\phi$ from a tropical curve $\Gamma$ to a space $X$ with an abstract rational polyhedral complex structure $\Sigma$ is called integral affine if it is piece-wise linear with integral slopes in $X$. In other words, $\phi$ is integral affine if for any point $v$ of $\Gamma$ and any sufficiently small segment $I$ on an edge adjacent to $v$ whose image under $\phi$ entirely lies inside some $\tau$ in $\Sigma$, the image of $I$ in $|\tau|$ is a segment with slope in $N_{\tau, \mathbb{Q}}$.

The main result of this section is the following theorem, whose proof gives an explicit description of the behavior of the reduced divisor $D_{P} \sim D$ under infinitesimal changes of the base point $P$.

Theorem 3. Let $D$ be a divisor of non-negative rank $r(D) \geq 0$. The map Red : $\Gamma \rightarrow|D| \hookrightarrow \Gamma^{(\operatorname{deg}(D))}$ defined by sending a point $P \in \Gamma$ to the point defined in $\Gamma^{\operatorname{deg}(D)}$ by the (unique) $P$-reduced divisor $D_{P}$ linearly equivalent to $D$ is integral affine, and thus, continuous.

Proof. Let $P$ be a point of $\Gamma$ and $E=D_{P}$ be the unique $P$-reduced divisor linearly equivalent to $D$. We give an explicit behavior of Red around $P$ which shows in particular that Red is integral affine.

Let $\vec{u}$ be a unit vector tangent to $\Gamma$ at $P$. (It is clear that there are $\operatorname{deg}(P)$ unit vectors tangent to $\Gamma$ at $P$.) We consider the interval $\left[P, P+\delta_{0} \vec{u}\right]$ for $\delta_{0}$ small enough and show how the family of reduced divisors $D_{v}$ behave for $v$ a point of $\Gamma$ in this interval. We first choose $\delta_{0}$ small enough such that there is no point of the support of $E\left(=D_{P}\right)$ in the half-open interval $\left(P, \delta_{0} \vec{u}\right]$. Since we will be dealing only with effective divisors, the first property of reduced divisors, namely the non-negativity of coefficients outside the base point, is obviously verified for the divisor $E$ and for every point in this interval. Thus, we will consider below the second property concerning closed connected subsets and show how the divisor $E$ has to be modified for any point of the interval $\left[P, P+\delta_{0} \vec{u}\right]$ in order to respect this property.

The following cases can happen:

(i) $E$ itself is $Q$-reduced for all the points $Q \in\left[P, \delta_{0} \vec{u}\right]$. (This case happens for example when the coefficient of $E$ at $P$ is zero, i.e., $E(P)=0$.)

In this case, the restriction of Red to $\left[P, P+\delta_{0} \vec{u}\right]$ is the constant map, i.e., Red contracts the interval $\left[P, P+\delta_{0} \vec{u}\right]$ to a point $E$. It is obvious that Red is integral affine restricted to this interval.

(ii) There are points $Q \in\left[P, \delta_{0} \vec{u}\right]$ for which $E$ is not $P$-reduced. (From the description we give below, it will be clear that, in this case, $E$ cannot be reduced for any point of the interval $\left(P, \delta_{0} \vec{u}\right]$.)

This means that there exists a closed connected set $X$ which does not contain $Q$ and such that for any point $v \in \partial X$, we have $E(v) \geq \operatorname{deg}_{X}^{\text {out }}(v)$. In this case $P$ should belong to $X$; otherwise the cut $X$ would violate the assumption that $E$ is $P$ reduced. By the choice of $\delta_{0}$, for all the points $v \in\left(P, P+\delta_{0} \vec{u}\right]$, we have $E(v)=0$. 
Since $X$ separates $P$ and $Q$ in the interval $[P, Q]$, we have $\left[P, P+\delta_{0} \vec{u}\right] \nsubseteq X$. We conclude that $X \cap\left[P, P+\delta_{0} \vec{u}\right]=P$. Thus, we have a closed connected set $X$ with the following properties:

(1) The intersection of $X$ with the interval $\left[P, P+\delta_{0} \vec{u}\right]$ contains only $P$.

(2) For all $v \in \partial X, E(v) \geq \operatorname{deg}_{X}^{\text {out }}(v)$.

In particular, this shows that $E$ is not reduced for any point of the interval $(P, P+$ $\left.\delta_{0} \vec{u}\right]$.

Let $\mathcal{F}$ be the family of all the closed connected subsets $X$ of $\Gamma$ with the above two properties. For any set $X \in \mathcal{F}$ and for any point $v \in \partial X$, we have $E(v) \geq$ $\operatorname{deg}_{X}^{\text {out }}(v) \geq 1$, which implies that $\mathcal{F}$ is a finite set. Define $Y$ as the union of all the elements of $\mathcal{F}, Y:=\bigcup_{X \in \mathcal{F}} X$. Next we will show that $Y$ itself is a closed connected subset of $\Gamma$ which verifies conditions (1) and (2) above. In other words, we will see that $Y$ is the maximal element of $\mathcal{F}$.

Property (1) easily follows by observing that $Y \cap\left[P, P+\delta_{0} \vec{u}\right]=\bigcup_{X \in \mathcal{F}}(X \cap$ $\left.\left[P, P+\delta_{0} \vec{u}\right]\right)=\{P\}$. To show property (2), let $v$ be a point of $\partial Y$. By the finiteness of $\mathcal{F}$, there exists at least one $X \in \mathcal{F} \operatorname{such}$ that $\operatorname{deg}_{Y}^{\text {out }}(v) \leq \operatorname{deg}_{X}^{\text {out }}(v)$. This clearly shows that $E(v) \geq \operatorname{deg}_{Y}^{\text {out }}(v)$.

Let $\delta_{0}>0$ be a small enough positive real such that for any point $v \in \partial Y$ and any unit vector $\vec{w}$ tangent to $\Gamma$ at $v$ and pointing outside $Y$, the whole interval $\left(v, v+\delta_{0} \vec{w}\right]$ lives outside $Y$ and does not contain any point of the support of $E$.

Let $\vec{w}_{1}, \ldots, \vec{w}_{s}$ different from $\vec{u}$ be all the different unit vectors tangent to $\Gamma$ at points $v_{1}, \ldots, v_{s} \in \partial Y$, respectively, and pointing out of $Y$. Remark that we might have $v_{i}=v_{j}$ for two different indices $i$ and $j$. The intervals $\left(v_{i}, v_{i}+\delta_{0} \vec{w}_{i}\right]$ together with $\left[P, P+\delta_{0} \vec{u}\right]$ form (a segment on) all the out-going edges from $Y$.

To simplify the presentation below, define the excess of $E$ at $P$ with respect to the cut $Y$ to be the integer quantity $\operatorname{ex}_{E, Y}(P):=E(P)-\operatorname{deg}_{Y}^{\text {out }}(P)+1$. Note that since $P$ is a boundary point of $Y$, and $Y$ verifies property (2), we have $\operatorname{ex}_{E, Y}(P) \geq 1$.

For any non-negative $\delta \leq \frac{\delta_{0}}{\operatorname{ex}_{E, Y}(P)}$, the rational function $f_{\delta}: \Gamma \rightarrow \mathbb{R}$ is defined as follows:

- the restriction of $f_{\delta}$ on $Y$ is zero,

- the restriction of $f_{\delta}$ on any interval $\left[v_{i}, v_{i}+\operatorname{ex}_{E, Y}(P) . \delta \vec{w}_{i}\right]$ is linear of slope -1 for any $1 \leq i \leq s$,

- the restriction of $f_{\delta}$ on the interval $[P, P+\delta \vec{u}]$ is linear of slope $-\operatorname{ex}_{E, Y}(P)$, and

- $f_{\delta}$ takes value $-\delta \cdot \operatorname{ex}_{E, Y}(P)$ at any other point of $\Gamma$.

Obviously, by the properties of $Y$, the divisor $E+\operatorname{div}\left(f_{\delta}\right)$ is effective. For any $\delta \leq \frac{\delta_{0}}{\operatorname{ex}_{E, Y}(P)}$ define the point $Q_{\delta}$ of $\Gamma$ by $Q_{\delta}=P+\delta \vec{u}$. We claim that $E+\operatorname{div}\left(f_{\delta}\right)$ is $Q_{\delta}$-reduced. This will in turn imply the theorem. Indeed, assuming the claim, it is fairly easy to check that $E+\operatorname{div}\left(f_{\delta}\right)$ is integral affine on the interval $\left[0, \frac{\delta_{0}}{\operatorname{ex}_{E, Y}(P)}\right]$. More explicitly, the image by the reduced divisor map Red of any point $Q_{\delta}$ in the

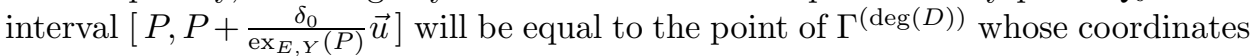
are described as follows. Among the $\operatorname{deg}(D)$ points of $\Gamma$ in $\operatorname{Red}\left(Q_{\delta}\right)$,

- $\operatorname{ex}_{E, Y}(P)$ of them are equal to $Q_{\delta}=P+\delta \vec{u}$,

- $s$ of them are the points $v_{1}+\delta \cdot \operatorname{ex}_{E, Y}(P) \vec{w}_{i}, \ldots, v_{s}+\delta \cdot \operatorname{ex}_{E, Y}(P) \vec{w}_{s}$, and 
- the remaining points are given by the image

$$
\operatorname{Red}\left(\sum_{v \neq P}\left(E(v)-\operatorname{deg}_{Y}^{\text {out }}(v)\right)(v)\right)
$$

in $\Gamma^{\left(\operatorname{deg}(D)-s-\operatorname{ex}_{E, Y}(P)\right)}$.

(Here $\operatorname{deg}_{Y}^{\text {out }}(v)=0$ if $v$ is not a boundary point of $Y$.) Since $\operatorname{Red}\left(\sum_{v \neq P}(E(v)-\right.$ $\left.\left.\operatorname{deg}_{Y}^{\text {out }}(v)\right)(v)\right)$ does not depend on $\delta$ and so is a constant function on the interval $\left[0, \frac{\delta_{0}}{\operatorname{ex}_{E, Y}(P)}\right]$, and since the other points are integral affine functions of $\delta$, the whole map Red becomes integral affine on the interval $\left[P, P+\frac{\delta_{0}}{\operatorname{ex}_{E, Y}(P)} \vec{u}\right]$. This being true for any $P$ and any tangent vector $\vec{u}$ to $\Gamma$ at $P$, the statement of the theorem follows.

Thus, all we need to prove this is to show that $E^{\delta}:=E+\operatorname{div}\left(f_{\delta}\right)$ is $Q_{\delta}$-reduced. For the sake of a contradiction, suppose this is not the case. Since $E^{\delta}$ is effective, this means there exists a closed connected subset $X$ of $\Gamma$ which does not contain $Q_{\delta}$ and such that for all $v$ on the boundary of $X$, we have $E^{\delta}(v) \geq \operatorname{deg}_{X}^{\text {out }}(v)$. First we claim that $X$ does not contain $P$, and more generally any point of the interval $\left[P, Q_{\delta}\right]$. Indeed, otherwise, since $X$ does not contain $Q_{\delta}$, it should contain a point $v$ of $\left[P, Q_{\delta}\right)$ on its boundary. But clearly for this point we have $E^{\delta}(v)=0<\operatorname{deg}_{X}^{\text {out }}(v)$, which is a contradiction by the choice of $X$. We now show that $X$ contains one of the points $v_{i}+\delta \cdot \operatorname{ex}_{E, Y}(P) \vec{w}_{i}$ on its boundary. Indeed, since $E$ is $P$-reduced and $X$ is a closed and connected subset which does not contain $P$, by the definition of reduced divisors there exists a point $v \in \partial X$ such that $E(v)<\operatorname{deg}_{X}^{\text {out }}(v)$. If $v \neq v_{i}+\delta \cdot \operatorname{ex}_{E, Y}(P) \vec{w}_{i}$ for all $1 \leq i \leq s$, then by the construction of $E^{\delta}$ we have $E^{\delta}(v) \leq E(v)<\operatorname{deg}_{X}^{\text {out }}(v)$, which again leads to a contradiction by our choice of $X$. This shows our second claim.

To summarize, we have proved so far that $X$ is a closed connected subset of $\Gamma$ which does not contain any point of $\left[P, Q_{\delta}\right]$, while $X$ contains at least one of the points $v_{i}+\delta \cdot \operatorname{ex}_{E, Y}(P) \vec{w}_{i}$ on its boundary $\partial X$. Without loss of generality, let $v_{1}+\delta \cdot \operatorname{ex}_{E, Y}(P) \vec{w}_{1}, \ldots, v_{k}+\delta \cdot \operatorname{ex}_{E, Y}(P) \vec{w}_{k}, k \geq 1$, be all the points among $v_{i}+\delta \vec{w}_{i}$ for $i \leq s$ which lie on the boundary of $X$.

For any point $v_{i}+\delta \cdot \operatorname{ex}_{E, Y}(P) \vec{w}_{i}$ in $\partial X$, we claim that either $\left[v_{i}, v_{i}+\delta \cdot \operatorname{ex}_{E, Y}(P) \vec{w}_{i}\right]$ $\subset X$ or $\left(v_{i}, v_{i}+\delta \cdot \operatorname{ex}_{E, Y}(P) \vec{w}_{i}\right] \cap X=\left\{v_{i}+\delta \cdot \operatorname{ex}_{E, Y}(P) \vec{w}_{i}\right\}$. To see this, note that if none of these situations happen, then $X$ has to contain another point $x$ of $\left(v_{i}, v_{i}+\delta \cdot \operatorname{ex}_{E, Y}(P) \vec{w}_{i}\right]$ on its boundary. For this point, obviously, we have $E^{\delta}(x)=$ $0<\operatorname{deg}_{X}^{\text {out }}(x)$, which is certainly a contradiction by the choice of $X$. Thus, without loss of generality, assume $v_{1}+\delta \cdot \operatorname{ex}_{E, Y}(P) \vec{w}_{1}, \ldots, v_{h}+\delta \cdot \operatorname{ex}_{E, Y}(P) \vec{w}_{h}, h \leq k$, are all the points on the boundary of $X$ with the property that

$$
\left(v_{i}, v_{i}+\delta \cdot \operatorname{ex}_{E, Y}(P) \vec{w}_{i}\right] \cap X=\left\{v_{i}+\delta \cdot \operatorname{ex}_{E, Y}(P) \vec{w}_{i}\right\} .
$$

We divide the rest of the proof into two cases, depending on whether $h=0$ or $h \geq 1$.

- $h=0$; in other words, for any $i \leq k,\left[v_{i}, v_{i}+\delta \cdot \operatorname{ex}_{E, Y}(P) \vec{w}_{i}\right] \subset X$.

Let $X^{\prime}:=X \backslash \bigcup_{i \leq k}\left(v_{i}, v_{i}+\delta \cdot \operatorname{ex}_{E, Y}(P) \vec{w}_{i}\right]$. Clearly $X^{\prime}$ is a closed connected subset of $\Gamma$ which does not contain $P$. Since $E$ is $P$-reduced, there exists a point $v \in \partial X^{\prime}$ such that $E(v)<\operatorname{deg}_{X^{\prime}}^{\text {out }}(v)$. We claim that there exists a $j \leq k$ such that $v=v_{j}$. Indeed, if $v \neq v_{j}$ for all $j \leq k$, then $E^{\delta}(v) \leq E(v)<\operatorname{deg}_{X^{\prime}}^{\text {out }}(v)=\operatorname{deg}_{X}^{\text {out }}(v)$, which clearly contradicts the assumption we made on $X$. Now consider the set $J$ of all indices $j \leq k$ such that $v_{j}=v$. Obviously, we have $\operatorname{deg}_{X^{\prime}}^{\text {out }}(v)=\operatorname{deg}_{X}^{\text {out }}(v)+|J|$. By 
the definition of $E^{\delta}$, we also have $E^{\delta}(v) \leq E(v)-|J|$. It follows that $E^{\delta}(v) \leq E(v)-|J|<\operatorname{deg}_{X^{\prime}}^{\text {out }}(v)-|J|=\operatorname{deg}_{X}^{\text {out }}(v)$. Since $E^{\delta}$ is effective, we infer that $v \in \partial X$ and $E^{\delta}(v)<\operatorname{deg}_{X}^{\text {out }}(v)$. This is again in contradiction with our assumption on $X$. Thus, $E^{\delta}$ is $Q_{\delta}$-reduced in this case.

- $h \geq 1$.

In this case, define

$$
Y^{\prime}=(Y \cup X) \backslash \bigcup_{h+1 \leq i \leq k}\left(v_{i}, v_{i}+\delta \cdot \operatorname{ex}_{E, Y}(P) \vec{w}_{i}\right]
$$

Since $h \geq 1$, we obviously have $Y \subsetneq Y^{\prime}$. Obviously, $Y^{\prime}$ is closed and connected, and contains $P$. We will show that for all the points $v \in \partial Y^{\prime}$, we have $E(v) \geq \operatorname{deg}_{Y^{\prime}}^{\text {out }}(v)$; in other words, $Y^{\prime} \in \mathcal{F}$ (for the family $\mathcal{F}$ defined at the beginning of the proof). This will be in contradiction with the maximality of $Y$ in $\mathcal{F}$, and thus finishes the proof of the theorem. Let $v \in \partial Y^{\prime}$. Two cases can occur:

Either, $v \in\left\{v_{1}, \ldots, v_{k}, P\right\}$, in which case, by the properties of $Y$ and the fact that $Y \subset Y^{\prime}$, we have

$$
E(v) \geq \operatorname{deg}_{Y}^{\text {out }}(v) \geq \operatorname{deg}_{Y^{\prime}}^{\text {out }}(v) .
$$

Or, $v \in \partial X \backslash\left\{v_{i}+\delta \cdot \operatorname{ex}_{E, Y}(P) \vec{w}_{i}\right\}_{i=1}^{k}$ and $v$ is not equal to any of the points $P, Q_{\delta}$, and $v_{i}$, for $i \leq k$. In this case, by the choice of $X$, we have $E^{\delta}(v) \geq \operatorname{deg}_{X}^{\text {out }}(v)$.

By the definition of $E^{\delta}$, we have $E(v)=E^{\delta}(v)$. We again infer that

$$
E(v) \geq \operatorname{deg}_{X}^{\text {out }}(v) \geq \operatorname{deg}_{Y^{\prime}}^{\text {out }}(v) .
$$

\section{Ample Divisors AND CANONICAL EMBEDDINGS}

In the previous section, we proved that the map $\operatorname{Red}_{D}: \Gamma \rightarrow|D|$ is continuous. By analogy with the classical case, in this section we consider the cases where the map defines an embedding of the tropical curve. Since $|D|$ does not admit a tropical structure in general, here by an embedding we simply mean an injective (proper) map. The following definition could be thought of as the tropical analogue of ample and very ample divisors on algebraic curves, via embeddings into projective spaces (see [6]).

Recall that $R(D)$ denotes the set of all rational functions $f$ on $\Gamma$ such that $D+\operatorname{div}(f) \geq 0$.

Definition 4 (Ample and very ample divisors). A divisor $D$ is called very ample if the rational functions in $R(D)$ separate points of $\Gamma$, i.e., for all pairs of points $P \neq Q$ in $\Gamma$, there exist two rational functions $f, g \in R(D)$ such that $f(P)-g(P) \neq$ $f(Q)-g(Q)$. A divisor $D$ is called ample if an integer multiple of $D$ is very ample, i.e., if there exists $m \in \mathbb{N}$ such that $m D$ is very ample.

Remark 5. If $D$ itself is an effective divisor, i.e., if $0 \in R(D)$, the assertion that $D$ is very ample is equivalent to the existence of a function $f \in R(D)$ such that $f(P) \neq f(Q)$.

We have the following tropical analogue of the classical theorem on characterization of very ample divisors in terms of embeddings into linear systems (for curves). 
Theorem 6. A divisor $D$ is very ample if and only if the map Red defines an embedding of $\Gamma$ in $|D|$.

The proof is based on the following lemma.

Lemma 7. Let $P \in \Gamma$ and $D$ be a P-reduced divisor. A function $f \in R(D)$ takes its maximum value at $P$.

Proof. For the sake of a contradiction suppose this is not the case and let $X$ be the set of all points $Q \in \Gamma$, where $f$ takes its maximum (thus, $P \notin X$ ). Note that $X$ is a closed subset of $\Gamma$. Since $f \in R(D)$, we have $D+\operatorname{div}(f) \geq 0$. Since $D$ is $P$-reduced and $X$ is closed and does not contain $P$, there is point $v \in \partial X$ such that $\operatorname{deg}_{X}^{\text {out }}(v)>D(v)$. Note that $f$ is strictly decreasing along any outgoing branch of $X$ at $v$, so the coefficient of $v$ in $D+\operatorname{div}(f)$ is at most $D_{v}-\operatorname{deg}_{X}^{\text {out }}(v)<0$. This contradicts the assumption that $D+\operatorname{div}(f)$ is effective, and the lemma follows.

Proof of Theorem 6. We show that $\operatorname{Red}_{D}$ is injective if and only if $D$ is very ample.

- Let $D$ be very ample. For the sake of a contradiction, suppose that $\operatorname{Red}_{D}$ is not injective. This means there are two points $P$ and $Q$ such that the $P$ - and $Q$-reduced divisors linearly equivalent to $D$ are the same, i.e., $D_{P}=D_{Q}$. For any rational function $f \in R\left(D_{P}\right)=R\left(D_{Q}\right)$, by applying Lemma 7 we know that $f$ takes its maximum value both at $P$ and $Q$. This means that $f(P)=f(Q)$ for all $f \in R\left(D_{P}\right)$, and this contradicts the assumption that $D$ is a very ample divisor (see Remark 5).

- If $\operatorname{Red}_{D}$ is injective, then $D_{P} \neq D_{Q}$. For two points $P \neq Q$ in $\Gamma$, there exists a non-constant rational function $g \in R\left(D_{P}\right)$ such that $D_{P}+\operatorname{div}(g)=D_{Q}$. By Lemma 7 applied twice, $g$ takes its maximum value at $P$ and $-g$ takes its maximum value at $Q$. Since $g$ is not constant, this means that $g(P) \neq g(Q)$, and so $D$ is very ample.

Corollary 8. If $\operatorname{deg}(D) \geq 2 g+1$, then $D$ is very ample. In particular, a divisor $D$ is ample if and only if $\operatorname{deg}(D)>0$.

Proof. This is a consequence of Theorem [6 Let $E=D_{P} \sim D$ be $P$-reduced. By the Riemann-Roch theorem, $E$ is effective. We claim that the coefficient of $P$ in $E$ is at least $\operatorname{deg}(D)-g$. Consider the set $S$ obtained as the union of all the branching points of $\Gamma$ and all the points in the support of $E$ :

$$
S:=\operatorname{support}(E) \cup\{\text { branching points of } \Gamma\} .
$$

Suppose $|S|=N+1$ and greedily define an ordering $v_{0}, \ldots, v_{N}$ of $S$ as follows. Start by setting $v_{0}=P$. Suppose by induction that the partial ordering $v_{0}, \ldots, v_{i}$ is already defined. Consider $X_{i}$ to be the cut defined by all the points of $S \backslash$ $\left\{v_{0}, \ldots, v_{i}\right\}$. This is basically obtained by taking all the segments joining two points of $S \backslash\left\{v_{0}, \ldots, v_{i}\right\}$ in $\Gamma$. Since $P \notin X_{i}$ and $E$ is $P$-reduced, there exists a point $v$ such that $E(v)<\operatorname{deg}_{X_{i}}^{\text {out }}(v)$. Define $v_{i+1}=v$. It is straightforward to check that $\sum_{i \geq 1} \operatorname{deg}_{X_{i}}^{\text {out }}\left(v_{i+1}\right)-1=g$. Thus, by the construction of the ordering, we have

$$
\sum_{i \geq 1} E\left(v_{i}\right) \leq \sum_{i \geq 1} \operatorname{deg}_{X_{i}}^{\text {out }}\left(v_{i+1}\right)-1=g .
$$

This clearly implies that $E(P)=E\left(v_{0}\right)=\operatorname{deg}(D)-\sum_{i \geq 1} E\left(v_{i}\right) \geq \operatorname{deg}(D)-g$, which establishes the claim. 
To conclude the proof, observe that if $\operatorname{deg}(D) \geq 2 g+1$, it cannot happen that $D_{P}=D_{Q}$. Otherwise, we would have

$$
\begin{aligned}
\operatorname{deg}(D) \geq D_{P}(P)+D_{P}(Q) & =D_{P}(P)+D_{Q}(Q) \\
& \geq \operatorname{deg}(D)-g+\operatorname{deg}(D)-g \geq \operatorname{deg}(D)+1 .
\end{aligned}
$$

By Theorem 6. $D$ is very ample provided that $\operatorname{deg}(D) \geq 2 g+1$.

We end this section by giving an improvement on the statement of Corollary 8 , First, we note that the bound $\operatorname{deg}(D) \geq 2 g+1$ in the above corollary is tight. To give an example, consider a tropical curve $\Gamma_{g}$ obtained by associating positive lengths to the edges of the banana graph of genus $g$ with two branching points $P$ and $Q$ of degree $g+1$ connected by $g+1$ parallel edges; see Figure $1(\mathrm{a})$. The divisor $D=g(P)+g(Q)$ on $\Gamma_{g}$ has degree $2 g$ and is both $P$ - and $Q$-reduced, so $D$ is not very ample. However, this is the only situation where this happens. More precisely, if $\Gamma$ does not have any model whose underlying graph is isomorphic to the banana graph of genus $g$, i.e., the graph underlying $\Gamma_{g}$, then every divisor $D$ of degree at least $2 g$ is very ample. We show this by reduction to absurdity, so for the sake of a contradiction, consider a divisor $E$ of degree $2 g$ which is both $P$-and $Q$-reduced for two different points $P \neq Q$ of $\Gamma$. As the proof of Corollary 8 shows, the coefficients of $P$ and $Q$ in $E$ are at least $g$, and since $\operatorname{deg}(E)=2 g$ and $E$ is effective, then we must have $E=g(P)+g(Q)$. Since $E$ is $P$-reduced, by taking the cut consisting of the only point $Q$, we see that $\operatorname{deg}_{\Gamma}(Q) \geq g+1$. A similar argument shows that $Q$ is not a cut vertex, i.e., $\Gamma \backslash\{Q\}$ is connected. Similarly $\operatorname{deg}_{\Gamma}(P) \geq g+1$ and $P$ is not a cut vertex. The genus of $\Gamma$ being $g$, we infer that $\operatorname{deg}_{\Gamma}(P)=\operatorname{deg}_{\Gamma}(Q)=g+1$ and all the other points are of degree two. Since none of $P$ and $Q$ is a cut vertex, this shows that the underlying graph of $\Gamma$ consists of $P$ and $Q$ and $g+1$ parallel edges between them.

3.1. Canonical embedding. In this section, we consider the $\operatorname{map} \operatorname{Red}_{K}$ defined by the canonical divisor $K=K_{\Gamma}$ of a tropical curve $\Gamma$. We provide a complete characterization of all the situations where the canonical divisor is not very ample.

We start this section by proving the following simple lemma.

Lemma 9. Let $\Gamma$ be a tropical curve of genus $g \geq 2$. If $K$ is not very ample, then there are two points $P$ and $Q$ of $\Gamma$ such that $K_{P}=K_{Q}=(g-1) \cdot(P)+(g-1) .(Q)$. Here $K_{P}$ and $K_{Q}$ are the $P$ - and $Q$-reduced divisors linearly equivalent to $K$.

Proof. We again use Theorem 6. If $K$ is not very ample, then the $\operatorname{map} \operatorname{Red}_{K}$ is not injective; i.e., there exist two points $P$ and $Q$ such that $K_{P}=K_{Q}$. We claim that the coefficient of $P$ in $K_{P}$ is at least $g-1$. Indeed, by the Riemann-Roch theorem, $K-(g-1)(P)$ has non-negative rank. This means that the $P$-reduced divisor associated to $K-(g-1)(P)$ is effective. This $P$-reduced divisor being simply $K_{P}-(g-1)(P)$, we infer that the coefficient of $P$ in $K_{P}$ is at least $g-1$. The same argument gives $K_{P}(Q)=K_{Q}(Q) \geq g-1$. Since $\operatorname{deg}(K)=2 g-2$ and all the other coefficients of $K_{P}$ are non-negative, we must have $K_{P}=K_{Q}=$ $(g-1) \cdot(P)+(g-1) \cdot(Q)$.

The following theorem characterizes all the situations where the canonical divisor is not very ample. We can assume that $\Gamma$ does not have any leaf.

Theorem 10. Let $\Gamma$ be a tropical curve of genus $g \geq 2$ without points of degree one. The only cases where the canonical divisor is not very ample are the following: 
C.I The underlying graph of $\Gamma$ consists of two vertices $P$ and $Q$ of degree $g+1$ which are connected by $g+1$ parallel edges. In particular, $K=(g-1)(P)+$ $(g-1)(Q)$.

C.II The underlying graph of $\Gamma$ consists of two vertices $P$ and $Q$ of degree $g$ which are joined by $g$ parallel edges and a point of degree four in the middle of one of the edges with an extra edge from this point to itself, i.e., a point $R$ such that the segments $[P, R]$ and $[R, Q]$ have the same length and there is a loop connecting $R$ to $R$ (see Figure $1(\mathrm{~b})$ ).

C.II' The underlying graph of $\Gamma$ consists of four branching points $P, Q, R$, and $S$ as in Figure $1(\mathrm{c})$, with $\operatorname{deg}(P)=\operatorname{deg}(Q)=g$ and $\operatorname{deg}(R)=\operatorname{deg}(S)=3$. In addition $[P, R]$ and $[Q, S]$ have the same length.

C.III The underlying graphs of $\Gamma$ have four branching points $P, Q, R$, and $S$ as in Figure $1(\mathrm{~d})$, with $\operatorname{deg}(P)=\operatorname{deg}(Q)=g$ and $\operatorname{deg}(R)=\operatorname{deg}(S)=3$. The lengths are arbitrary.

Recall that a tropical curve is called hyperelliptic if there exists a divisor $D$ of degree two such that $r(D)=1$. For a tropical curve whose canonical divisor is not very ample, let $D=(P)+(Q)$ such that $K \sim(g-1)$. $D$ by Lemma 9 , By the above characterization theorem, it is easy to see that $r(D)=1$. Since $\operatorname{deg}(D)=2$, the tropical curves $\Gamma$ appearing in the characterization theorem above are all hyperelliptic. In addition, one obtains that canonical divisors of generic tropical curves of genus $g \geq 2$ are very ample.

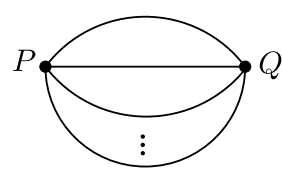

(a)

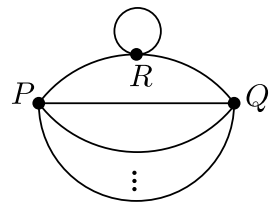

(b)

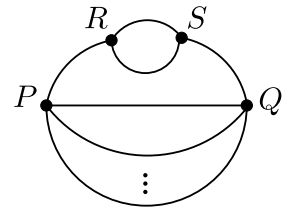

(c)

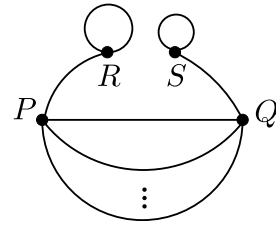

(d)

Figure 1. Cases where $K$ is not very ample; cf. Theorem 10 .

(a) Case C.I: $\operatorname{deg}(P)=\operatorname{deg}(Q)=g+1$, and the lengths are arbitrary.

(b) Case C.II: $\operatorname{deg}(P)=\operatorname{deg}(Q)=g$, $\operatorname{deg}(R)=4$, and $\ell([P, R])=$ $\ell([R, Q])$. The other lengths are arbitrary.

(c) Case C.II': $\operatorname{deg}(P)=\operatorname{deg}(Q)=g$, $\operatorname{deg}(R)=\operatorname{deg}(S)=3$, and $\ell([P, R])=\ell([S, Q])$. The other lengths are arbitrary.

(d) Case C.III: $\operatorname{deg}(P)=\operatorname{deg}(Q)=g$, $\operatorname{deg}(R)=\operatorname{deg}(S)=3$, and the lengths are arbitrary.

Proof of Theorem 10, Suppose $K$ is not very ample. By Lemma 9, there are two points $P$ and $Q$ such that $K_{P}=K_{Q}=(g-1)(P)+(g-1)(Q)$. Since $K_{P}$ is $P$ reduced, we have $\operatorname{deg}(Q) \geq g$. Similarly we have $\operatorname{deg}(P) \geq g$. Since $\sum_{P \in \Gamma} \operatorname{deg}(P)-$ $2=2 g-2$ and $\Gamma$ does not have any leaf, the following cases happen:

- $\operatorname{deg}(P)=\operatorname{deg}(Q)=g+1$. In this case, $K_{P}=K_{Q}$ since $P$-(resp. $\left.Q\right)$ reduced, $Q$ (resp. $P$ ) cannot be a cut vertex in $\Gamma$. Thus, the underlying graph of $\Gamma$ consists of two vertices $P$ and $Q$ of degree $g+1$ which are 


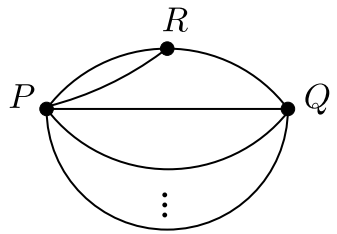

(a)

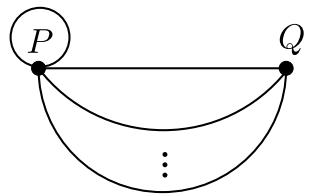

(b)

Figure 2. (a) $\operatorname{deg}(P)=g+1, \operatorname{deg}(Q)=g, \operatorname{deg}(R)=3$.

(b) $\operatorname{deg}(P)=g+2, \operatorname{deg}(Q)=g$.

connected by $g+1$ parallel edges. In particular, $K=(g-1)(P)+(g-1)(Q)$, and this is case C.I of the theorem.

- Either $\operatorname{deg}(P)=g+1$ and $\operatorname{deg}(Q)=g$, or $\operatorname{deg}(P)=g$ and $\operatorname{deg}(Q)=g+1$. Without loss of generality, suppose that $\operatorname{deg}(P)=g+1$ and $\operatorname{deg}(Q)=g$. In this case, $\Gamma$ has a branching point $R \neq P, Q$ of degree three. The underlying graph of $\Gamma$ is the graph of Figure $2(\mathrm{a})$. It is easy to see that since $R \neq Q$, $K \nsim(g-1)(P)+(g-1)(Q)$, so this situation cannot happen.

- $\operatorname{deg}(P)=\operatorname{deg}(Q)=g$. In this case, $\Gamma$ has either one branching point $R \neq P, Q$ of degree four, or two different branching points $R \neq P, Q$ and $S \neq P, Q$ of degree three. The underlying graph of $\Gamma$ then has a very particular shape. A case analysis shows that the only possibilities for $(g-$ $1)(P)+(g-1)(Q)$ to be equivalent to the canonical divisor are the cases C.II, C.II', and C.III described in the theorem; see Figures $1(\mathrm{~b}) 1(\mathrm{c}) 1(\mathrm{~d})$.

- $\operatorname{deg}(P)=g$ and $\operatorname{deg}(Q)=g+2(\operatorname{or} \operatorname{deg}(P)=g+2$ and $\operatorname{deg}(Q)=g)$. In this case, the underlying graph of $\Gamma$ consists of the two points $P$ and $Q$ joined by $g$ parallel edges and there is an extra loop based on $P$ (see Figure 2(b)]. It is easy to show that this case cannot happen.

The theorem follows.

\section{Applications: Tropical Weierstrass points AND RANK-DETERMINING SETS}

We already saw some applications of the reduced divisor map, especially in obtaining a characterization of all the tropical curves with a very ample canonical divisor. In this section, we provide two more applications of this method: we first provide an effective way of proving the existence of Weierstrass points on tropical curves of genus at least two, and then proceed by giving an alternative shorter proof of a theorem of Luo on tropical rank-determining sets.

4.1. Tropical Weierstrass points. Let $\Gamma$ be a tropical curve of genus $g$, and $K$ the canonical divisor of $\Gamma$. By analogy with the theory of algebraic curves, we say that $P \in \Gamma$ is a Weierstrass point if $r(g(P)) \geq 1$. Equivalently, by the RiemannRoch theorem, a point $P \in \Gamma$ is Weierstrass if and only if $r(K-g(P)) \geq 0$, i.e., if $K-g(P)$ is equivalent to an effective divisor. The Riemann-Roch theorem also implies

Proposition 11. For every point $P \in \Gamma, r(K-(g-1)(P)) \geq 0$. Let $K_{P}=$ $a_{P}(P)+\sum_{v: v \neq p} a_{v}(v)$ be the (unique) P-reduced divisor linearly equivalent to $K$. Then $a_{P} \geq g-1$. In addition, $P$ is Weierstrass if and only if $a_{P} \geq g$. 
In this section we show the following tropical analogue of the classical theorem that every smooth projective curve of genus at least two over an algebraically closed field $\kappa$ has a Weierstrass point.

Theorem 12. Any tropical curve of genus at least two has a Weierstrass point.

Before going through the proof, the following remarks are in order.

Remark 13. - The theorem easily follows by Baker's specialization lemma [2], by using the existence of a degeneration of smooth curves to $\Gamma$, from the classical theorem on the existence of Weierstrass points; see [2] for more details. The proof given here has the advantage of being more explicit, providing an efficient way of finding a Weierstrass point.

- In the classical setting, it is possible to count the number of Weierstrass points in a very precise sense; see Section 6. In the tropical context, such a formula cannot exist, at least with the definition of Weierstrass points given above. Indeed, as was pointed out in [2], the example of the graph $\Gamma_{g}$ with two branching points of degree $g+1$ with $g+1$ parallel edges for $g \geq 3$ (Figure 1(a) shows that $\Gamma$ can have an infinite number of such points. In this tropical curve, it is quite easy to see that there exists an interval of positive length on each edge joining $P$ to $Q$ whose points are all Weierstrass.

Proof of Theorem 12, Let $\Gamma$ be a tropical curve of genus $g \geq 2$ and $K$ the canonical divisor of $\Gamma$. Let Red : $\Gamma \rightarrow \Gamma^{(2 g-2)}$ be the map defined in Section 2 which sends a point $P \in \Gamma$ to the point of $\Gamma^{(2 g-2)}$ which corresponds to the unique $P$-reduced divisor $K_{P}$ linearly equivalent to $K$. Our proof will essentially be based on Theorem 3 and the explicit description of the behavior of the map Red given in the proof of Theorem 3 .

Suppose for the sake of a contradiction that there is no Weierstrass point in $\Gamma$. Then, by Proposition 11, for every point $P \in \Gamma$, the coefficient of $P$ in $K_{P}$ is exactly $g-1$. Let $F: \Gamma \rightarrow \mathbb{R}_{\geq 0}$ be the continuous function defined as follows. For a point $P \in \Gamma$, let $P_{1}, \ldots, P_{g-1}$ be all the points in the support of $K_{P}$ which are different from $P$ (potentially we could have $P_{i}=P_{j}$ for some $i \neq j$ ). The value of $F$ at $P$ is defined as $F(P):=\min \left\{\operatorname{dist}_{\Gamma}\left(P, P_{i}\right)\right\}_{i=1}^{g-1}$. Recall that $\operatorname{dist}_{\Gamma}(.,$.$) denotes the$ distance between points in $\Gamma$. By Theorem 3, the point $\left(P_{1}, \ldots, P_{g-1}\right) \in \Gamma^{(g-1)}$ is a continuous function of $P$, thus the map $F$ is continuous. By the compactness of $\Gamma$ and continuity of $F$, there is a point $P \in \Gamma$ such that $F$ takes its minimum value at $P$, i.e., $F(P)$ is the minimum of $F(Q)$ over $Q \in \Gamma$. By our assumption, all the points $P_{i}$ are different from $P$, and so $F(P)>0$. Let $P_{1} \neq P$ be the point in the support of $K_{P}$ such that $F(P)=\operatorname{dist}_{\Gamma}\left(P, P_{1}\right)$ and let $C$ be a shortest path from $P$ to $P_{1}$. We show that moving $P$ to another point $P^{\prime}$ in the direction of the shortest path $C$ will decrease the value of $F$, which will obviously be a contradiction.

Let $\vec{u}$ be the unit tangent vector to $\Gamma$ at $P$ such that $[P, P+\delta \vec{u}] \subset C$ for all sufficiently small $\delta$. We look at the behavior of $K_{P}$ in the interval $[P, P+\delta \vec{u}]$. Since for a point $Q$ in this interval the coefficient of $K_{Q}$ at $Q$ is at least $g-1 \geq 1, K_{P}$ is evidently not $Q$-reduced for $\delta$ small enough. Thus, let $Y$ be the maximal closed connected subset of $\Gamma$, defined in the proof of Theorem 3 such that

1. The intersection of $Y$ with the interval $[P, P+\delta \vec{u}]$ contains only $P$.

2. For all $v \in \partial Y, K_{P}(v) \geq \operatorname{deg}_{Y}^{\text {out }}(v)$. 
Note that, by our assumption, we also have $K_{P}(P)=g-1$. The proof of Theorem 3 shows that for $\delta$ small enough and for $Q \in[P, P+\delta \vec{u}]$, the coefficient of $K_{Q}$ at $Q$ is equal to $K_{P}(P)-\operatorname{deg}_{Y}^{\text {out }}(P)+1$. Since $K_{P}(P)=K_{Q}(Q)=g-1$, we must have $\operatorname{deg}_{Y}^{\text {out }}(P)=1$.

Two cases can happen:

- Either $P_{1} \notin Y$, in which case $K_{P}\left(P_{1}\right)=K_{Q}\left(P_{1}\right)$. In other words, $P_{1}$ lies in the support of $K_{Q}$ for all $Q \in[P, P+\delta \vec{u}]$ for $\delta>0$ small enough. However, note that $F(Q) \leq \operatorname{dist}_{\Gamma}\left(Q, P_{1}\right)<$ length $(C)=F(P)$, which leads to a contradiction.

- Or, $P_{1} \in Y$. We claim that in this case $P_{1} \in \partial Y$ and $C \cap Y=\left\{P_{1}, P\right\}$. Suppose for the sake of a contradiction that this is not true, i.e., $P_{1}$ lies in the interior of $Y$ or $C$ intersects $Y$ in a point different from $P_{1}$ and $P$. Since $C$ is a path from $P$ to $P_{1}$ and since the interval $[P, P+\delta \vec{u}] \subset C$ and $(P, P+\delta \vec{u}] \cap Y=\emptyset$, in either of these cases there must exist a point $v \in \partial Y$, $v \neq P_{1}, P$, which is the first time $C$ returns to $Y$ while going from $P$ to $P_{1}$. By property 2 of the set $Y$ above, we have $K_{P}(v) \geq \operatorname{deg}_{Y}^{\text {out }}(v) \geq 1$, i.e., $v$ lies in the support of $K_{P}$. We have $\operatorname{dist}_{\Gamma}(P, v)<\operatorname{dist}_{\Gamma}\left(P, P_{1}\right)=F(P)$, which contradicts the definition of $F$. This proves our claim.

Let $\vec{w}$ be the unit vector tangent to $\Gamma$ at $P_{1}$ such that the interval $\left[P_{1}, P_{1}+\right.$ $\delta \vec{w}$ ] lies inside $C$ for $\delta$ small enough. By our explicit description of the reduced divisors $K_{Q}$ for $Q \in[P, P+\delta \vec{u}]$, given in the proof of Theorem 3 , there is a point $Q_{1} \in\left[P_{1}, P_{1}+\delta \vec{w}\right]$ such that the coefficient of $Q_{1}$ in $K_{Q}$ is one, provided that $Q$ is close enough to $P$. This shows that $F(Q) \leq$ $\operatorname{dist}_{\Gamma}\left(Q, Q_{1}\right)<\operatorname{length}(C)=F(P)$, which again leads to a contradiction by our choice of the point $P$.

The proof of Theorem 12 is now complete.

The exact same argument gives the following more general statement.

Theorem 14. Let $D$ be a divisor of positive rank. If for all $P \in \Gamma$ the coefficient of $D_{P}$ at $P$ is at least $a$, for some integer $a \geq 1$, then there exists a point $P \in \Gamma$ such that the coefficient of $P$ in $D_{P}$ is at least $a+1$.

Definition 15. Let $D$ be a divisor of rank $r$. A point $P \in \Gamma$ is called a Weierstrass point for $D$ (or a $D$-Weierstrass point) if the coefficient of $D_{P}$ at $P$ is at least $r+1$.

Corollary 16. Let $\Gamma$ be a tropical curve and $D$ be a divisor of positive rank. Then $\Gamma$ has a D-Weierstrass point.

Proof. Let $r=r(D) \geq 1$. By definition of rank, for any point $Q \in \Gamma$, the coefficient of $Q$ in $D_{Q}$ is at least $r$. By Theorem 14, there exists a point $P \in \Gamma$ with $D_{P}(P) \geq$ $r+1$, i.e., $P$ is a $D$-Weierstrass point.

It is worth noticing that Remark 13 still remains valid for $D$-Weierstrass points. It would be interesting to have a definition of tropical Wronskians (as in the classical setting; cf. Section 6) which would allow one to obtain more precise statements about the number of such points.

4.2. Rank-determining sets of points. Recall that a subset $A \subset \Gamma$ is called rank-determining 8] if the rank of a divisor in $\Gamma$ is equal to the maximum $r$ such that for any effective divisor $E$ of degree $r$ with support in $A$, the divisor $D-E$ has non-negative rank (equivalently, $D-E$ is equivalent to an effective divisor). 
The following proposition can be easily proved by induction on $r$.

Proposition 17. A given subset $A \subset \Gamma$ is rank-determining if and only if for a divisor $D$ of non-negative rank the following two assertions are equivalent.

- Rank of $D$ is at least one, $r(D) \geq 1$.

- For any point $P \in A, P$ is in the support of $D_{P}$.

Let $A$ be a set of points of $\Gamma$. By cutting $\Gamma$ along $A$ we mean the (possibly disconnected) tropical curve obtained as the disjoint union of the closure in $\Gamma$ of each of the connected components of $\Gamma \backslash A$.

Theorem 18 (Luo [8]). Given a subset $A \subset \Gamma$, if cutting $\Gamma$ along the points of $A$ results in a disjoint union of at least two tropical curves and if all the connected components of the cutting are of genus zero, then $A$ is rank-determining.

Before giving the proof, the following remarks are in order.

Remark 19. (1) For a model $G=(V, E)$ of a tropical curve $\Gamma$ without loops, the vertices of $V$ form a rank-determining set. This was essentially proved in [7.

(2) Luo's theorem is a more general statement in terms of special open sets (see 8 for the definition) and gives a necessary and sufficient condition for a subset $A$ of $\Gamma$ to be rank-determining. We restrict ourselves to the above statement; however, we note that the proof given below works without any change in that more general setting and derives the sufficiency of the condition in Luo's theorem.

Proof of Theorem 18. Using Proposition 17 (twice, once for $A$ and once for $\Gamma$ ), we need to prove that if $P$ belongs to the support of $D_{P}$ for every point $P$ in $A$ and if cutting $\Gamma$ along $A$ results in a set of at least two tropical rational curves (i.e., of genus zero), then for any point $Q \in \Gamma, Q$ is in the support of $D_{Q}$. Let $\Gamma_{1}, \ldots, \Gamma_{s}$ be all the tropical curves obtained by cutting $\Gamma$ along $A$, so $s \geq 2$. Without loss of generality, we can assume that $Q \in \Gamma_{1}$. Let $\tilde{\Gamma}_{1} \subset \Gamma_{1}$ be the set of all the points $P$ of $\Gamma$ such that $P$ lies in the support of $D_{P}$. For the sake of a contradiction, suppose that $Q \notin \tilde{\Gamma}_{1}$. Let $X$ be the closure in $\Gamma$ of the connected component of $\Gamma_{1} \backslash \tilde{\Gamma}_{1}$ which contains $Q$. Note that $X$ is a rational tropical curve, and for all the points $P$ of the boundary of $X$ in $\Gamma, P$ belongs to the support of $D_{P}$ (by the continuity of Red). However, for all the points $P$ in the interior of $X, P$ does not belong to the support of $D_{P}$. We prove the following claim:

Let $P$ be a point on the boundary of $X$. Then the $P$-reduced divisor $D_{P}$ is reduced for all the points of $X$. In particular, all the points of the boundary of $X$ belong to the support of $D_{P}$ and no interior point lies in the support.

This will certainly be a contradiction: for the point $Q$ in the interior of $X$, the cut $\Gamma \backslash \operatorname{int}(X)$ is saturated. Indeed, all the points on the boundary of this cut have valence one, and the coefficient of $D_{Q}$ at these points is at least one since by the above claim all the points belong to the support of $D_{Q}$.

To prove the claim, we use the explicit description of the reduced divisor map. First of all, again as above, for any point $P$ on the boundary of $X$, there is a unique vector $\vec{v}$ emanating from $P$ in the direction of $X$. By the description of the reduced divisor in the interval $[P, P+\epsilon \vec{v}], D_{P}$ is $(P+\epsilon \vec{v})$-reduced for all $\epsilon$ small enough. 
Indeed, if this was not the case, then $P+\epsilon \vec{v}$ would belong to the support of $D_{P+\epsilon \vec{v}}$, which is certainly not true by the definition of $X$. For the sake of a contradiction, suppose now that the claim does not hold. Then there exists a point $R$ in the interior of $X$ and a direction $\vec{v}$ emanating from $R$ such that $D_{R}=D_{P}$ (for the boundary point $P$ of $X$ as above) and such that $D_{P}$ is not reduced for any point $R+\epsilon \vec{v}$, for $\epsilon$ sufficiently small. In this case, $R$ should be in the support of $D_{R}$. Indeed, as we saw in the proof of Theorem 3, if the coefficient of $R$ in $D_{R}$ was zero, then $D_{R}$ would remain obviously reduced for all the points sufficiently close to $R$. This is not the situation here, and so $D_{R}(R) \geq 1$. But this is again a contradiction by the choice of $X$ : no point $R$ in the interior of $X$ can belong to the support of $D_{R}$. Since $X$ is connected and Red is continuous, this shows that all the reduced divisors $D_{R}$, for $R \in X$, are equal to $D_{P}$, and the claim follows. This completes the proof of the theorem.

\section{Maps into tropical PROJECTIVE SPACES}

In this section, we give some complementary results on the image of the map Red and relate this map to maps into tropical projective spaces.

Let $D$ be a divisor on a tropical curve $\Gamma$. Let $R(D)$ be the space of all rational functions $f$ such that $\operatorname{div}(f)+D \geq 0$. Since two rational functions $f$ and $g$ have the same divisor if and only if $f=c \odot g$ for a constant $c \in \mathbb{T}$, we have $|D|=\mathbb{P} R(D):=$ $R(D) / \mathbb{T}$; the quotient is obtained by identifying $f$ with $c \odot f$.

5.1. The tropical module structure of $R(D)$. For two rational functions $f$ and $g$, the function $f \oplus g$ is a rational function on $\Gamma$ (recall that $(f \oplus g)(x):=$ $\max \{f(x), g(x)\})$. In addition, it is quite easy to show that $\operatorname{div}(f \oplus g)+D \geq 0$. It follows that $R(D)$ admits the structure of a tropical module over $\mathbb{T}$, the semi-field of tropical numbers. (Recall that the tropical multiplication $c \odot f$, for $c \in \mathbb{T}$ and $f \in R(D)$, is defined by $(c \odot f)(x):=f(x)+c$. $)$

Let $D$ be a divisor of non-negative rank. For a point $P$ in $\Gamma$, let $f_{P}$ be the unique rational function in $R(D)$ such that

(i) $D+\operatorname{div}\left(f_{P}\right)=D_{P}$, the unique $P$-reduced divisor equivalent to $D$.

(ii) $f_{P}(P)=0$.

The fact that $f_{P}$ is unique comes from the unicity of $D_{P}$ and the assumption on the value of $f_{P}$ at $P$. Below we show a set of properties of the rational functions $f_{P}$. Some complement properties are given in Section 5.4 below.

The first lemma gives a precise characterization of the rational function $f_{P}$. For a set of functions $S=\{h: \Gamma \rightarrow \mathbb{T}\}$, the tropical sum $\bigoplus_{h \in S} h$ is the function defined by $x \rightarrow \max _{h \in S} h(x)$ for all $x \in \Gamma$.

Lemma 20. Let $D$ be a divisor of non-negative rank on a tropical curve and $f_{P}$ be the rational function defined above. We have

$$
f_{P}=\bigoplus_{h \in R(D): h(P)=0} h .
$$

Proof. Since $f_{P}$ itself appears in the tropical sum on the right hand side of the above equation, we only need to show that for all $Q \in \Gamma$ and $h \in R(D)$ with $h(P)=0$, we have $f_{P}(Q) \geq h(Q)$. By Lemma [7, we know that $h-f_{P}$ takes its maximum at $P$. This proves the lemma since this shows that for all $Q \in \Gamma$, $h(Q)-f_{P}(Q) \leq h(P)-f_{P}(P)=0$. 
Remark 21. As a tropical module, $R(D)$ cannot be generated in general by the set $\left\{f_{P}\right\}$ for $P \in \Gamma$. Indeed, for $g \in R(D)$ the existence of a solution $\left\{x_{P} ; P \in \Gamma\right\}$ for the equation $g=\bigoplus_{P \in \Gamma} x_{P} \odot f_{P}$ (or, equivalently, for the family of equations $g(Q)=\max _{P \in \Gamma} x_{P}+f_{P}(Q)$ for all $\left.Q\right)$ can be equivalently described as follows. First note that the unique solution of the above equations, if it exists, is given by $x_{P}=\min _{Q \in \Gamma} g(Q)-f_{P}(Q)$, and the following claim has to be true:

if $S_{P}$ denotes the set of all points $Q$ such that $x_{P}=g(Q)-f_{P}(Q)$, then

$$
\bigcup_{P \in \Gamma} S_{P}=\Gamma \text {. }
$$

Let $E=\operatorname{div}(g)+D=\operatorname{div}\left(g-f_{P}\right)+\operatorname{div}\left(f_{P}\right)+D=\operatorname{div}\left(g-f_{P}\right)+D_{P}$. If $Q \in S_{P}$ and if $g \neq c \odot f_{P}$ for all $P \in \Gamma$ and $c \in \mathbb{T}$, then $g-f_{P}$ takes at least two values, and it takes its minimum at $Q$, so for every point $v$ on the boundary of $S_{P}$, we have $E(v) \geq \operatorname{deg}_{S_{P}}^{\text {out }}(v)$. Call a cut $X$ good for $E$ if for all points $v$ on the boundary of $X$, we have $E(v) \geq \operatorname{deg}_{X}^{\text {out }}(v)$. As we just noticed, the existence of a solution $\left\{x_{P} ; P \in \Gamma\right\}$ implies that all the cuts $S_{P}$ are good for $E$ and cover the entire $\Gamma$. However, there exist divisors which are not reduced for any point of $\Gamma$ and for which the union of the good cuts is not $\Gamma$. For example, consider a tropical curve with two branching points of degree three and three parallel edges between them. Let $P$ and $Q$ be two points in the interior of two different edges. The divisor $E=2 P+2 Q$ is not reduced for any point of $\Gamma$, and the only cuts $X$ which are good for $E$ are $\{P\}$, $\{Q\}$, and $\{P, Q\}$. We do not know the answer to the following question: provided that the union of all the good cuts for $E$ is $\Gamma$, is it true that $g$ can be written as $g=\bigoplus_{P \in \Gamma} x_{P} \odot f_{P}$ for some $x_{P}$ 's in $\mathbb{T}$ ?

5.2. On the image of the reduced divisor map. In this section we consider the image of the tropical curve $\Gamma$ under the map Red and study the basic properties of this map. We then relate our map Red to maps from $\Gamma$ to tropical projective spaces considered in [6].

We start by recalling the following basic result from [6] (see the discussion in Section 2.1).

Proposition 22 (Hasse, Muskier, and $\mathrm{Yu}[6]$ ). Let $D^{\prime}$ be an element of $|D|$ and $X$ be the set of all the points of the support of $D^{\prime}$ which lie in the interior of an edge of $\Gamma$. The dimension of the cell of $|D|$ which contains $D^{\prime}$ is one less than the number of connected components of $\Gamma \backslash X$.

Corollary 23. The image of Red lies in the 1-skeleton of the cell complex $|D|$.

Proof. Let $E=D_{P}$ be the $P$-reduced divisor equivalent to $D$. Let $X$ be the set of all points in the support of $E$ which lie in the interior of an edge. Two cases can happen:

- $P \notin X$. In this case we claim that $\Gamma \backslash X$ is connected, i.e., $E$ is a vertex of the cell complex $|D|$. Indeed, if this is not the case, then by taking a connected component of $\Gamma \backslash X$ which does not contain the point $P$, we obtain a cut in which all the boundary points are in the interior of an edge and lie in the support of $E$. This clearly contradicts the assumption that $E$ is $P$-reduced.

- $P \in X$. We claim that $\Gamma \backslash X$ contains either one or two connected components. If this was not the case, then there would exist a connected component of $\Gamma \backslash X$ which does not contain $P$, and we again obtain a contradiction to the assumption that $E$ is $P$-reduced. 
This combined with Proposition 22 above clearly completes the proof.

Theorem 24. Let $\Gamma$ be a tropical curve. Every point in the image of Red is either a vertex of $|D|$ or a tropical convex combination of two vertices of $|D|$ which are in the image of the map Red. The tropical convex hull of the image of Red is finitely generated.

Proof. Let $T$ be the set of all the points $D_{Q}$ which are among the vertices of $|D|$. We first claim that the tropical convex hull of the image of Red is generated by the set of all $D_{Q} \in T$. For this aim, we will only need to show that for any point $P \in \Gamma$, the $P$-reduced divisor $D_{P} \sim D$ can be written as a convex hull of two elements of $T$. Let $P \in \Gamma$. If $D_{P} \in T$, we do not have anything to prove. So suppose that $D_{P} \notin T$. This means that the set $X$ consisting of all the points in the support of $D_{P}$ which lie in the interior of an edge contains the point $P$ and $\Gamma \backslash X$ consists of two connected components. Let $\vec{u}$ and $\vec{w}$ be the two unit vectors tangent to $\Gamma$ at $P$. By the explicit description of the reduced divisor map we gave in Section 22 it is easy to see that $f_{P}$ can be written as the tropical convex hull of the rational functions $f_{P+\epsilon_{0} \vec{u}}$ and $f_{P+\delta_{0} \vec{w}}$, where $\epsilon_{0}$ and $\delta_{0}$ are, respectively, the supremum value of $\epsilon$ and $\delta$ such that for all the values of $\epsilon^{\prime}$ in $[0, \epsilon]$ and $\delta^{\prime}$ in $[0, \delta]$, the support points of the corresponding reduced divisors living in the interior of the edges form a cut. The two divisors $D_{P+\epsilon_{0} \vec{u}}$ and $D_{P+\delta_{0} \vec{w}}$ are certainly vertices of $|D|$. We conclude that every point in the image of Red is a tropical convex combination of two vertices of $|D|$ which lie in the image of Red. This also shows that the tropical convex hull of the image of Red is a finitely generated module.

We define the reduced linear system of $D$ as follows.

Definition 25. Define $\tilde{R}(D)$ to be the tropical convex hull of the image of Red, i.e., the tropical module generated by the rational functions $f_{P}$. The reduced linear system of $D$, denoted by $|D|_{r}$, is defined to be $\tilde{R}(D) / \sim$.

Contrary to what one might expect, it is not in general true that the tropical convex hull of the image of Red has dimension equal to $r(D)$. An example is given by a tropical curve $\Gamma$ consisting of two branching points of degree three $P$ and $Q$ connected with three parallel edges. The divisor $2 P+Q$ has rank one, while the tropical convex hull of the image of Red has dimension two.

5.3. Maps to tropical projective spaces. Given a fixed model for $\Gamma$, by Theorem 24, $\tilde{R}(D)$ is generated by all the vertices of $|D|$ which are reduced with respect to some base point in $\Gamma$. Let $\mathcal{F}$ be the set of all the rational functions $f_{P}$ corresponding to these vertices. $\mathcal{F}$ allows one to define a map from $\Gamma$ to $\mathbb{T P}\left(\mathcal{F}^{*}\right)$. This latter space is the tropical projective space consisting of all the maps $\mathcal{F} \rightarrow \mathbb{T}$ modulo tropical multiplication by a constant. Note that the map $\phi_{\mathcal{F}}: \Gamma \rightarrow \mathbb{T P}\left(\mathcal{F}^{*}\right)$ is canonical once a model for $\Gamma$ is fixed. In particular, if $\Gamma$ is of genus at least two and the model of $\Gamma$ consists of the coarsest model (i.e., all the vertices are the branching points), then the map $\Gamma \rightarrow \mathbb{T} \mathbb{P}\left(\mathcal{F}^{*}\right)$ is canonical.

The following theorem establishes a precise relation between our map Red and the embeddings of tropical curves into tropical projective spaces considered in [6].

Theorem 26. $\quad$ 1. The image of $\phi_{\mathcal{F}}$ is homeomorphic to the image of Red.

2. Also, there is an injective map $|D|_{r} \rightarrow \mathbb{T P}\left(\mathcal{F}^{*}\right)$ with image trop-conv $\left(\phi_{\mathcal{F}}(\Gamma)\right)$, the tropical convex hull of the image of $\phi_{\mathcal{F}}$ in $\mathbb{T} \mathbb{P}\left(\mathcal{F}^{*}\right)$. In particular, $|D|_{r}$ is 
homeomorphic to trop-conv $\left(\phi_{\mathcal{F}}(\Gamma)\right)$. In other words, there exists a commutative diagram

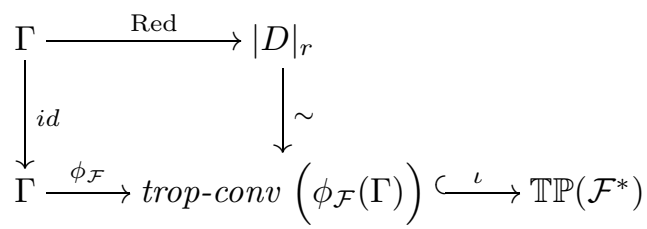

Proof. 1. Define a map $\Psi: \phi_{\mathcal{F}}(\Gamma) \rightarrow|D|_{r}=\tilde{R}(D) / \sim$ as follows. Given a point $P \in \Gamma$, let $\Psi\left(\phi_{\mathcal{F}}(P)\right)=\bigoplus_{f \in \mathcal{F}}(-f(P)) \odot f$. We claim that $\Psi\left(\phi_{\mathcal{F}}(P)\right)=f_{P}$ in $\tilde{R}(D)$. From the description we give below, this will prove the first part of the theorem. To show this, note that since $\mathcal{F}$ generates $\tilde{R}(D)$ as a tropical module, there are $x_{f} \in \mathbb{T}$ for $f \in \mathcal{F}$ such that $\bigoplus_{f \in \mathcal{F}} x_{f} \odot f=f_{P}$. This means that $x_{f}+f(P) \leq f_{P}(P)=0$ for all $f \in \mathcal{F}$ or, equivalently $x_{f} \leq-f(P)$. In particular, we must have $f_{P}=\bigoplus_{f \in \mathcal{F}} x_{f} \odot f \leq \Psi\left(\phi_{\mathcal{F}}(P)\right)$. Since $(-f(P)) \odot f$ takes value zero at $P$, by applying Lemma 20 we have $f_{P}=\Psi\left(\phi_{\mathcal{F}}(P)\right)$, and the claim follows. To prove the first part, note that there is a map from $\operatorname{Red}(\Gamma) \rightarrow \phi_{\mathcal{F}}(\Gamma)$ obtained by sending $f_{P}$ to $\phi_{\mathcal{F}}(P)$. This map is well defined since $f_{P}=\bigoplus_{f \in \mathcal{F}} x_{f} \odot f$ and $-f(P)$ is the maximum value $x_{f}$ can take while verifying the above equation. Thus, for two points $P$ and $Q$ with $f_{P}=f_{Q}$, we have $f(P)=f(Q)$ for all $f \in \mathcal{F}$.

2. Define a map $\Theta:|D|_{r} \rightarrow \mathbb{T P}\left(\mathcal{F}^{*}\right)$ as follows. Given an element $g \in|D|_{r}$, $\Theta(g) \in \mathbb{T} \mathbb{P}\left(\mathcal{F}^{*}\right)$ is the map which sends $f \in \mathcal{F}$ to $\max _{Q \in \Gamma} f(Q)-g(Q)$ :

$$
\Theta(g): \mathcal{F} \rightarrow \mathbb{T} \text { is given by } \Theta(g)(f)=\max _{Q \in \Gamma} f(Q)-g(Q) \text {. }
$$

As mentioned in Remark 21, an element $g \in|D|_{r}=\operatorname{trop}$-conv $\left(\left\{f_{P}\right\}_{P \in \Gamma}\right)$ can be written in the form $g=\bigoplus_{f \in F} x_{f} \odot f$, where $x_{f}:=\min _{Q \in \Gamma} g(Q)-f(Q)$, so that the map $\Theta(g)$ sends $f$ to $-x_{f}$. By definition, it is clear that $\Theta(\lambda \odot g)=(-\lambda) \odot \Theta(f)$ so that $\Theta$ is a well-defined map from $|D|_{r}$ to $\mathbb{T P}\left(\mathcal{F}^{*}\right)$. Note also that the restriction of $\Theta$ on $\operatorname{Red}(\Gamma)$ is the inverse of the map $\Psi$ constructed above. Indeed, if $g=f_{P}$ for a point $P \in \Gamma$, then the rational function $f-f_{P}$ takes its maximum value at $P$, and this value is equal to $f(P)$. Thus, $\Theta\left(f_{P}\right)=\phi_{\mathcal{F}}(P)$. In addition, the image of $\Theta$ clearly lies in the tropical convex hull of the image of $\phi_{\mathcal{F}}$. By the very definition, the injectivity of $\Theta$ follows. To show that $\Theta$ is surjective, let $\eta: \mathcal{F} \rightarrow \mathbb{R}$ be a given map sending $f$ to $\eta_{f}$ and $\eta=\bigoplus_{Q \in \Gamma} h(Q) \odot \phi_{\mathcal{F}}(Q)$. Define the rational function $g$ in $|D|_{r}$ as the tropical sum $g:=\bigoplus_{f \in \mathcal{F}}\left(-\eta_{f}\right) \odot f$. We claim that $\Theta(g)=\eta$.

By assumption, we have $\eta_{f}=\max _{Q} h(Q)+f(Q)$. Thus, $-h(Q) \geq f(Q)-\eta_{f}$ for all $f$, i.e.,

$$
-h(Q) \geq g(Q), \quad \forall Q \in \Gamma .
$$

Note also that by the definition of $g$, we have for fixed $f$,

$$
\eta_{f} \geq f(Q)-g(Q) \quad \forall Q \in \Gamma .
$$

Since $\eta_{f}=\max _{Q \in \Gamma} h(Q)+f(Q)$, there is a $Q_{0}$ such that $\eta_{f}=h\left(Q_{0}\right)+f\left(Q_{0}\right)$. For this particular point $Q_{0}$, the above inequality becomes

$$
h\left(Q_{0}\right)+f\left(Q_{0}\right) \geq f\left(Q_{0}\right)-g\left(Q_{0}\right), \quad \text { i.e., } \quad-h\left(Q_{0}\right) \leq g\left(Q_{0}\right) .
$$


We infer that $h\left(Q_{0}\right)=-g\left(Q_{0}\right)$, and $\eta_{f}=f\left(Q_{0}\right)-g\left(Q_{0}\right)$.

This shows that for all $f$, there is a point $Q$ such that $-h(Q)=g(Q)=f(Q)-\eta_{f}$, or $\eta_{f}=f(Q)-g(Q)=\max _{Q} f(Q)-g(Q)=\Theta(g)(f)$.

Corollary 27. Let $\mathcal{F}$ be defined as above. Then $D$ is very ample if and only if $\phi_{\mathcal{F}}$ is injective.

5.4. Duality. Let $f_{P}$ be as before the rational function defining the $P$-reduced divisor $D_{P}$, i.e., $D_{P}=D+\operatorname{div}\left(f_{P}\right)$, and $f_{P}(P)=0$. Consider the function $f_{Q}^{t}$ obtained from the family of rational functions $f_{P}$ by interchanging the role of base points and arguments, namely, $f_{Q}^{t}$ is the function defined by $f_{Q}^{t}(P)=-f_{P}(Q)$. We now show that all the functions $f_{Q}^{t}$ are rational and their tropical convex hull contains the tropical module $R(D)$. (Note that $f_{Q}^{t}$ does not necessarily lie in $R(D)$.)

To show the second assertion, note that for a point $P \in \Gamma$, we have $g(P) \odot$ $f_{P}^{t}(P)=g(P)+0=g(P)$, so we only need to show that for all $P$ and $Q$ in $\Gamma$, $g(P) \odot f_{P}^{t}(Q) \leq g(Q)$, or $g(P)-f_{Q}(P) \leq g(Q)-f_{Q}(Q)$. This directly follows from the fact that $g-f_{Q}$ takes its maximum value at $Q$; cf. Lemma 7 .

The fact that $f_{Q}^{t}$ is a rational function is an easy consequence of the results of the previous sections. Below, we give a more explicit proof which provides a description of the sectional derivatives of $f_{Q}^{t}$ in terms of the results of Section 2.2, For fixed $Q \in \Gamma$, and for any $P \in \Gamma$ and any unit vector $\vec{u}$ emanating from $P$, we need to calculate $\left.\frac{d}{d \epsilon} f_{Q}^{t}(P+\epsilon \vec{u})\right|_{\epsilon=0}$ and show that it is an integer. By definition, this is $-\left.\frac{d}{d \epsilon} f_{P+\epsilon \vec{u}}(Q)\right|_{\epsilon=0}=\lim _{\epsilon \rightarrow 0} \frac{-f_{P+\epsilon \vec{u}}(Q)+f_{P}(Q)}{\epsilon}$. Note that $g_{\epsilon}()=.f_{P+\epsilon \vec{u}}()-.f_{P}($. satisfies $D_{P}+\operatorname{div}\left(g_{\epsilon}\right)=D_{P+\epsilon \vec{u}}$. This shows that $g_{\epsilon}$ is the rational function given in the proof of Theorem 3 up to an additive constant. The constant is defined by the requirement that $f_{P+\epsilon \vec{u}}(P+\epsilon \vec{u})=0$. A simple calculation shows that the derivation is exactly

$$
-\left.\frac{d}{d \epsilon} f_{P+\epsilon \vec{u}}(Q)\right|_{\epsilon=0}=\frac{d}{d \epsilon} f_{P}(P+\epsilon \vec{u})-h_{\vec{u}}(Q),
$$

where the function $h_{\vec{u}}$ is defined as follows. Let $Y$ be the cut considered in the proof of Theorem 3 which contains $P$, does not contain $P+\epsilon \vec{u}$ and which is maximum with respect to the property that for all $v$ on its boundary, $D_{P}(v) \geq \operatorname{deg}_{Y}^{\text {out }}(v)$. If such a cut does not exist, simply let $Y=\emptyset$. Then $h_{\vec{u}}(Q)=0$ if $Q$ lies outside $Y$ and $h_{\vec{u}}(Q)=D_{P}(P)-\operatorname{deg}_{Y}^{\text {out }}(P)+1$ if $Q$ is in $Y$. In particular, the slope of $f_{Q}^{t}$ at $P$ is an integer. It is clear that the number of these slopes is finite. This shows that $f_{Q}^{t}$ is a rational function. By the description of the directional derivatives of $f_{Q}^{t}$ at $P$, we also see that the coefficient of $D+\operatorname{div}\left(f_{Q}^{t}\right)$ at $P$ is $D_{P}(P)-\sum_{\vec{u}} h_{\vec{u}}(Q)$, where the sum is over all the unit vectors $\vec{u}$ tangent to $\Gamma$ at $P$ and where $h_{\vec{u}}(Q)$ is either zero or equal to $D_{P}(P)-\operatorname{deg}_{Y}^{\text {out }}(P)+1$, depending on whether $Q$ lies outside or inside $Y$, respectively.

\section{Algebraic CURVes And the ReduCED Divisor MAP}

To make the analogy between the classical and the tropical case, in this section we provide the classical analogue of the reduced-divisor map.

Let $C$ be a non-singular projective curve over an algebraically closed field $\kappa$ of characteristic zero. Let $D$ be a divisor on $C$ and $P$ be a point of $C$. A divisor $D^{\prime} \sim D$ is called $P$-reduced if all the coefficients of $D^{\prime}$ outside $P$ are non-negative, 
and, in addition, the coefficient of $P$ in $D^{\prime}$ is maximum among all the divisors linearly equivalent to $D$ with this property.

Proposition 28. For every divisor $D$ on $C$, there exists a unique $P$-reduced divisor linearly equivalent to $D$.

Proof. The existence of divisors $D^{\prime} \sim D$ with non-negative coefficients outside $P$ is easy (e.g., is a direct consequence of Riemann-Roch). Since the coefficients of $D^{\prime}$ are bounded above by the degree of $D$, the existence of $P$-reduced divisors follows. To show the uniqueness, note that if $f$ and $g$ are two non-collinear rational functions such that both $D^{\prime}=D+\operatorname{div}(f)$ and $D^{\prime \prime}=D+\operatorname{div}(g)$ are $P$-reduced, and so have the same coefficient at $P$, then for two appropriately chosen non-zero scalars $\alpha$ and $\beta$ in $\kappa$, the non-zero rational function $\alpha f+\beta g$ has an order at $P$ strictly larger than that of $f$ and $g$. In addition, $D+\operatorname{div}(\alpha f+\beta g)$ has non-negative coefficients outside $P$. This leads to a contradiction, and the proposition follows.

Let $D$ be a divisor on $C$ of non-negative rank, and let $\mathcal{L}=\mathcal{L}(D)$ be the corresponding invertible sheaf on $C$. Let $L$ be the vector space of the global sections of $\mathcal{L}(D) ; L$ is the vector space of all the rational functions $f$ with $D+\operatorname{div}(f) \geq 0$ $(L=R(D)$ with our earlier notation). Note that $\operatorname{dim}(L)=r(D)+1$. Denote by $L^{*}$ the dual of $L$.

There are two maps from $C$ to a projective space defined by the above data that we now explain.

The one that is easier to define, which is the one usually considered, is the map $\phi: C \rightarrow \mathbb{P}\left(L^{*}\right): \phi(P)$ is the point of $\mathbb{P}\left(L^{*}\right)$ corresponding to the hyperplane of $L$ defined by all the rational functions $f \in L$ such that the coefficient of $P$ in $D+\operatorname{div}(f)$ is at least one. Equivalently, the map $\phi$ is the map given generically by evaluating at the points of $C$, i.e., generically, $\phi(P)$ is the linear form on $L$ defined by sending a rational function $f \in L$ to $f(P)$. If $D$ is base point free, $\phi$ is well defined globally. In any case, the map can be extended to the whole of $C$.

The second map is $\eta: C \rightarrow \mathbb{P}(L)$, defined by reduced divisors. Suppose that $D$ has non-negative rank. For every point $P \in C$, let $D_{P}$ be the unique $P$-reduced divisor linearly equivalent to $D$. Since $r(D) \geq 0$, we have $D_{P} \geq 0$. Let $f_{P}$ be a rational function in $L$ such that $D_{P}=D+\operatorname{div}\left(f_{P}\right)$. Note that modulo multiplication by a constant, $f_{P}$ is well defined. In other words, the line $\ell_{P}$ defined by $f_{P}$ is well defined in $L$. Define a map $\eta: C \rightarrow \mathbb{P}(L)$ by setting $\eta(P)=\ell_{P}$ for any point $P \in C$.

We will show below that $\eta$ is a morphism defined by a set of sections of $\mathcal{L}^{\otimes r} \Omega^{\otimes r(r-1) / 2}$, where $\Omega$ is the invertible sheaf of differentials on $C$ and $r=r(D)$. These sections are defined in terms of Wronskians.

6.1. Wronskians and the reduced divisor map. We first obtain a generic description of the map $\eta$. Let $\mathbb{K}=\mathbb{K}(C)$ be the field of rational functions on $C$. Choose a basis $f_{0}, \ldots, f_{r}$ of $L$ over $\kappa$ and let $\mathcal{F}=\left\{f_{0}, \ldots, f_{r}\right\}$. Let $\Omega$ be the invertible sheaf of differentials on $C$. Let $P$ be a point of $C$ and $\mathcal{O}_{P}$ be the local ring of the structural sheaf $\mathcal{O}_{C}$ at $P . \Omega_{P}$ is the module of $\kappa$-differentials of $\mathcal{O}_{P}$ and $d: \mathcal{O}_{O} \rightarrow \Omega_{P}$ is the derivation.

If $\tau$ denotes a local parameter at $P$, a basis for $\Omega_{P}$ is given by $d \tau$.

The $\mathcal{O}_{P}$-module $\mathcal{L}_{P}$ is free of rank one; suppose that it is generated by $g_{P} \in \mathcal{L}_{P}$.

For each $i \in\{0, \ldots, r\}$, there exists an element $f_{i, P} \in \mathcal{O}_{P}$ such that $f_{i \mid P}=$ $f_{i, P} g_{P}$. 
For each $i \in\{0, \ldots, r\}$, inductively define $f_{i, P}^{(j)} \in \mathcal{O}_{P}$ as follows. Let $f_{i, P}^{(0)}:=f_{i, P}$, and suppose that $f_{i, P}^{(j)} \in \mathcal{O}_{P}$ is already defined. Then $f_{i, P}^{(j+1)}:=d f_{i, P}^{(j)} / d \tau \in \mathcal{O}_{P}$ (i.e., $f_{i, P}^{(j+1)}$ is the function defined by $\left.d f_{i, P}^{(j)}=f_{i, P}^{(j+1)} d \tau\right)$.

The Wronskian of $f_{0}, \ldots, f_{r}$ at $P$, denoted by $\mathrm{Wr}_{\mathcal{F}, P}$, is now defined as

$$
\mathrm{Wr}_{\mathcal{F}, P}=\operatorname{det}\left(f_{i, P}^{(j)}\right)_{i, j=0}^{r} g_{P}^{r+1}(d \tau)^{r(r+1) / 2} \in \Gamma\left(\mathcal{O}_{P}, \mathcal{L}_{P}^{\otimes(r+1)} \Omega_{P}^{\otimes r(r+1) / 2}\right) .
$$

It is easy to show that the above (local) definition does not depend on the choice of the local parameter $\tau$, and glue them together to define a non-zero global section $\mathrm{Wr}_{\mathcal{F}}$ of $\mathcal{L}^{\otimes(r+1)} \Omega^{\otimes r(r+1) / 2}$, i.e., $\mathrm{Wr}_{\mathcal{F}} \in \Gamma\left(C, \mathcal{L}^{\otimes(r+1)} \Omega^{\otimes r(r+1) / 2}\right)$. The fact that $\mathrm{Wr}_{\mathcal{F}}$ is non-zero comes from the non-triviality of the $\operatorname{determinant} \operatorname{det}\left(f_{i, P}^{(j)}\right)_{i, j=0}^{r}$ for linearly independent $f_{i, P}$ 's. This latter claim can easily be seen by looking at the completion $\hat{\mathcal{O}}_{P} \sim \kappa[[\tau]]$ of $\mathcal{O}_{P}$ at the maximal ideal $m_{P}$ of $P$ and the map $\mathcal{O}_{P} \rightarrow \kappa[[\tau]]$. The image of $\mathrm{Wr}_{\mathcal{F}, P}$ in $\kappa[[\tau]]$ is non-zero; see for example [4 for a simple proof.

Note that in the discussion above we only used the linear independence of the sections $f_{i}$. Thus, it is clear that instead of choosing a basis for $L$, we can take any family $\mathcal{G}$ consisting of $s \leq r+1$ linearly independent elements of $L$ and define the Wronskian $\mathrm{Wr}_{\mathcal{G}}$ with respect to that family. The Wronskian $\mathrm{Wr}_{\mathcal{G}}$ will then be a non-zero global section of $\mathcal{L}^{\otimes s} \omega^{\otimes s(s-1) / 2}$.

For $i \in \mathcal{F}$, define $\mathcal{F}_{i}=\mathcal{F} \backslash\{i\}$ and consider the Wronskian

$$
\mathrm{Wr}_{\mathcal{F}_{i}} \in \Gamma\left(C, \mathcal{L}^{\otimes r} \Omega^{\otimes r(r-1) / 2}\right) .
$$

Locally at a point $P$, and in the terminology of the previous paragraph, $\mathrm{Wr}_{\mathcal{F}_{i}}$ is the maximal minor of the matrix $\left(f_{i, P}^{(j)}\right)_{i=0, \ldots, r, j=0, \ldots, r-1}$ obtained by deleting the $i^{\text {th }}$ column. The sections $\mathrm{Wr}_{F_{i}}$ satisfy the following equations:

$$
\begin{aligned}
\forall 0 \leq j \leq r-1 \quad & \sum_{i=0}^{r}(-1)^{i} \mathrm{Wr}_{\mathcal{F}_{i}, P} f_{i, P}^{(j)}=0 \quad \text { and } \\
& \sum_{i=0}^{r}(-1)^{i} \mathrm{Wr}_{\mathcal{F}_{i}, P} f_{i, P}^{(r)}=\operatorname{Wr}_{\mathcal{F}, P} .
\end{aligned}
$$

Since $\mathrm{Wr}_{\mathcal{F}} \neq 0$, by the definition of $f_{i, P}^{(j)}$, it follows that generically the rational function $\sum_{i}(-1)^{i} \mathrm{Wr}_{\mathcal{F}_{i}, P}(P) f_{i}$ is exactly the one defining the reduced divisor $D_{P}$, i.e., the line defined by $\sum_{i} \operatorname{Wr}_{\mathcal{F}_{i}, P}(P) f_{i}$ is $\ell_{P}$ (the line defined by the rational function $f_{P}$ with $D+\operatorname{div}\left(f_{P}\right) P$-reduced). Here $\operatorname{Wr}_{\mathcal{F}_{i}, P}(P)$ is the value of $\operatorname{Wr}_{\mathcal{F}_{i}, P}$ at $P$.

Indeed, to see this, write $f_{P}=\sum a_{i} f_{i}$ for $a_{i} \in \kappa$, and note that $f_{P}$ has a zero of order at least $s$ at $P$ if and only if all the derivations of order at most $s-1$ of $f_{P}$ at $P$ are zero, i.e., $\sum_{i} a_{i} f_{i, P}^{(j)}(P)=0$. Now use $\mathrm{Wr}_{F} \neq 0$ and equations (11) and (2) to obtain the claim.

We infer that, generically, the map $\eta$ coincides with the map to $\mathbb{P}(L)\left(\sim \mathbb{P}^{r}\right.$ with respect to the basis $\left.f_{0}, \ldots, f_{r}\right)$ defined by the sections $\left\{(-1)^{i} \mathrm{Wr}_{\mathcal{F}_{i}}\right\}_{i=0}^{r}$ of $\mathcal{L}^{\otimes r} \Omega^{\otimes r(r-1) / 2}$ i.e., $\eta$ sends the point $P$ to the point $\left[(-1)^{0} \mathrm{Wr}_{\mathcal{F}_{0}}(P): \cdots\right.$ : $\left.(-1)^{r} \mathrm{Wr}_{\mathcal{F}_{r}}(P)\right]$ of $\left.\mathbb{P}^{r}\right)$. Let $\overline{\mathrm{Wr}}$ be the extension of the map defined by the sections $\mathrm{Wr}_{\mathcal{F}_{i}}$ to the whole curve $C$. 
We now show

Theorem 29. The map $\eta$ defined by the reduced divisors coincides globally with the morphism $\overline{\mathrm{Wr}}$ defined by the sections $(-1)^{i} \mathrm{Wr}_{\mathcal{F}_{i}}$ of $\mathcal{L}^{\otimes r} \Omega^{\otimes r(r-1) / 2}, i=0, \ldots, r$.

Proof. We have already seen that $\eta$ generically coincides with $\overline{\mathrm{Wr}}$, e.g., for those points of $C$ for which the determinants appearing in the definition of the Wronskians $\mathrm{Wr}_{\mathcal{F}_{i}}$ are not all zero. For a point $P$ and $i \in\{0, \ldots, r\}$, let $A_{i, P}$ be the determinant in the definition of $\mathrm{Wr}_{\mathcal{F}_{i}}$, in the terminology of the previous lines:

$$
A_{i, P}=\operatorname{det}\left(f_{j, P}^{\left(j^{\prime}\right)}\right)_{j=0, \ldots, \hat{i}, \ldots, r}^{j^{\prime}=0, \ldots, r-1}
$$

We now show that the image by $\overline{\mathrm{Wr}}$ of a point $P$ for which all the determinants $A_{i, P}$ are zero is exactly $\eta(P)$. This will complete the proof of the theorem.

Let $P$ be such a point, and let $v \geq 1$ be the integer such that $A_{i, P}=\tau^{v} h_{i}$ for all $i$, and at least for one $i, h_{i}(P) \neq 0$. The image of $P$ by $\overline{\mathrm{Wr}}$ is the point $\left[h_{0}(P):-h_{1}(P): \cdots:(-1)^{r} h_{r}(P)\right]$ of $\mathbb{P}^{r}(=\mathbb{P}(L)$ in the basis $\mathcal{F})$. We have to show that $\sum(-1)^{i} h_{i}(P) f_{i, P}$ has the maximum order of zero at $P$.

By considering the injective map $\mathcal{O}_{P} \rightarrow \kappa[[\tau]]$, we can suppose that all the elements $f_{i, P}$ and $h_{i}$ are in $\kappa[[\tau]]$, and evaluating at $P$ consists in setting $\tau=0$.

Write $f_{i, P}=\sum_{s \geq 0} a_{i, s} \tau^{j}$, and consider the vectors $V_{s}=\left(a_{0, s}, \ldots, a_{r, s}\right)$. For $i=$ $1, \ldots, r$, let $s_{i}$ be the smallest index $s$ such that the span of the vectors $V_{0}, \ldots, V_{s_{i}}$ has rank $i$. In particular, the span of $V_{s_{1}}, \ldots, V_{s_{r}}$ has rank $r$. We now show that $h_{0}(P), \ldots, h_{r}(P)$ are a constant multiple of the maximal minors of the $r(r+1)$ matrix $M$ with lines equal to the vectors $V_{s_{1}}, \ldots, V_{s_{r}}$,

$$
M:=\left(\begin{array}{c}
V_{s_{1}} \\
\vdots \\
V_{s_{r}}
\end{array}\right)=\left(\begin{array}{cccc}
a_{s_{1}, 0} & a_{s_{1}, 1} & \cdots & a_{s_{1}, r} \\
\vdots & \vdots & & \vdots \\
a_{s_{r}, 0} & a_{s_{r}, 1} & \cdots & a_{s_{r}, r}
\end{array}\right)
$$

This will of course complete the proof. Indeed, if $\mu_{i}$ denotes the maximal minor obtained by deleting the $i^{\text {th }}$ column, then by the definition of $s_{r}$ at least one of the $\mu_{i}$ 's is non-zero. In addition, for any vector $V_{s}$

$$
\sum_{j=0}^{r}(-1)^{i} \mu_{i} a_{i, s}=0 \quad \text { if and only if } V_{s} \text { belongs to the span of } V_{s_{1}}, \ldots, V_{s_{r}}
$$

thus, $\sum_{i}(-1)^{i} \mu_{i} f_{i, P}$ has the maximum order of zero at $P$.

We claim that $v=s_{1}+\left(s_{2}-1\right)+\cdots+\left(s_{r}-r\right)$ and the coefficient of $t^{v}$ in $A_{i, P}$ is $\left(\prod_{i=1}^{r} \frac{s_{i} !}{i !}\right) \mu_{i}$ (this is trivially equivalent to $\left.h_{i}(0)=\left(\prod_{i=1}^{r} \frac{s_{i} !}{i !}\right) \mu_{i}\right)$. The claim is easily obtained by developing the determinant in the definition of $A_{i, P}$. For any $s$, let $V_{s}^{i}=\left(a_{s, 0}, \ldots, \hat{a}_{s, i}, \ldots, a_{s, r}\right)$ so that

$$
\mu_{i}=\operatorname{det}\left(\begin{array}{c}
V_{s_{1}}^{i} \\
\vdots \\
V_{s_{r}}^{i}
\end{array}\right)
$$


We have

$$
A_{i, P}=\operatorname{det}\left(f_{j, P}^{\left(j^{\prime}\right)}\right)_{j=0, \ldots, \hat{i}, \ldots, r}^{j^{\prime}=0, \ldots, r-1}=\operatorname{det}\left(\begin{array}{c}
\sum_{j_{1} \geq 0} \tau^{j_{1}} V_{j_{1}}^{i} \\
\vdots \\
\sum_{j_{i} \geq i-1} \frac{j_{i} !}{i !} \tau^{j_{i}-i+1} V_{j_{2}}^{i} \\
\vdots \\
\sum_{j_{1} \geq 0, j_{2} \geq 1, \ldots, j_{r} \geq r-1} \\
\sum_{j_{r} \geq r-1} \frac{j_{r} !}{r !} \tau^{j_{r}-r+1} V_{j_{r}}^{i}
\end{array}\right)
$$
$\sum_{i=1}^{r}\left(j_{i}-i+1\right)<\sum_{i=1}^{r}\left(s_{i}-i+1\right)$, and the coefficient of $\tau^{\sum_{i=1}^{r}\left(s_{i}-i+1\right)}$ is exactly $\prod_{i=1}^{r} \frac{s_{i} !}{i !} \mu_{i}$. The proof of the theorem is now complete.

Note that $D$-Weierstrass points are precisely the points in the support of the effective divisor defined by the global section $\mathrm{Wr}_{\mathcal{F}}$ of $\mathcal{L}^{\otimes(r+1)} \Omega^{\otimes r(r+1) / 2}$, and the multiplicity of a Weierstrass point is defined to be the coefficient of the point in this divisor. This is a simple consequence of the definition of $\mathrm{Wr}_{\mathcal{F}}$ and equations (1) and (2) above. The total number of $D$-Weierstrass points, counted with multiplicity, is given by the degree of the invertible sheaf $\mathcal{L}^{\otimes(r+1)} \Omega^{\otimes r(r+1) / 2}$ which is $(r+1) \operatorname{deg}(D)+r(r+1)(g-1)$.

\section{ACKNOWLEDGMENT}

Part of this work was done during a visit of the author to Charles University in Prague in 2008. The author is very grateful to Dan Kràl' and the members of KAM for their warm hospitality and great discussions. Special thanks go to Matt Baker for his interest in this work, for bringing the author's attention to the work of [6] related to the results of this paper, and to the work of [8], and for his many constructive suggestions and interesting comments on the first draft of this paper which helped to improve the presentation. Many thanks to Erwan Brugallé for several interesting and fruitful discussions, and for motivating the author to write these results. Special thanks go to Pooran Memari and Mathieu Desbrun for their help and support during the preparation of this paper.

\section{REFERENCES}

1. O. Amini and M. Manjunath, Riemann-Roch for sub-lattices of the root lattice $A_{n}$, Electron. J. Combin. 17 (2010), no. 1, Research Paper 124, 50 pp. MR2729373 (2012a:06009)

2. M. Baker, Specialization of Linear Systems From Curves to Graphs. With an appendix by B. Conrad, Algebra Number Theory 2 (2008), no. 6, 613-653. MR2448666 (2010a:14012) 
3. M. Baker and S. Norine, Riemann-Roch and Abel-Jacobi theory on a finite graph, Adv. Math. 215 (2007), no. 2, 766-788. MR2355607 (2008m:05167)

4. A. Bostan and P. Dumas, Wronskians and linear independence, Amer. Math. Monthly 117 (2010), no. 8, 722-727. MR2732247 (2011g:15003)

5. A. Gathmann and M. Kerber, Riemann-Roch Theorem in Tropical Geometry, Math. Z. 259 (2008), no. 1, 217-230. MR2377750 (2009a:14014)

6. C. Hasse, G. Musiker, and J. Yu, Linear Systems on Tropical Curves, Math. Z., to appear.

7. J. Hladký, D. Kràl', and S. Norine, Rank of divisors on tropical curves, J. Combin. Theory Ser. A, to appear.

8. Y. Luo, Rank-determining sets of metric graphs, Combin. Theory Ser. A. 118 (2011), no. 6, 1775-1793. MR2793609(2012d:05122)

9. G. Mikhalkin and I. Zharkov, Tropical curves, their Jacobians and theta functions, Curves and abelian varieties, Contemp. Math., vol. 465, Amer. Math. Soc., Providence, RI, 2008, pp. 203-230. MR2457739(2011c:14163)

CNRS, Département de mathématiques et applications, École Normale Supérieure, 45 Rue d'Ulm, 75230 Paris Cedex 05, France

E-mail address: oamini@math.ens.fr 